\title{
Vague Lies: \\ How to Advise Consumers When They Complain*
}

\author{
Mikhail Drugov ${ }^{\dagger}$ and Marta Troya-Martinez
}

May 6, 2012

\begin{abstract}
This paper analyzes the incentives of a seller to provide (un)biased and (im)precise advice about a complex product such as insurance, banking and telecommunication services. Misleading the buyers by biasing the advice upwards increases the revenues but also the expected fine imposed by the authority. Making the advice less precise does not affect the revenues in equilibrium but interferes with the authority's inference and affects the expected fine in a non-monotonic way. In particular, making the advice less precise makes it harder to convict the seller but increases the expected fine when the seller is found guilty. We find that, in the equilibrium, biasing the advice and making it noisier are complements; in particular, a higher buyers' heterogeneity, a stricter standard of proof employed by the authority and a larger share of credulous consumers make the advice more biased and less precise.
\end{abstract}

Keywords: Cheap Talk, Advice, Legal Procedure, Consumer Protection.

JEL Classification: D18, D8, K4, L1.

\section{Introduction}

A buyer with limited or no experience walks into a shop in order to buy a mobile phone and sign up for a calling plan. There are many models of mobiles and different calling

*This paper has been previously circulated under the title "Bias, Noise and Consumer Protection". We are grateful to Vicente Cuñat, Péter Eső, Natalia Fabra, Rosa Ferrer, Maria Goltsman, Martin Hellwig, Paul Klemperer, Marc Möller, David Myatt, Bent Nielsen and participants of the workshop "Advances in Industrial Organisation" (Vienna), IIOC 2010 (Vancouver), CRESSE 2010 (Chania), JEI 2010 (Madrid) and the seminars at the Max Planck Institute for Research on Collective Goods (Bonn) and IIES (Stockholm) for many insightful comments. Any remaining errors are our own.

$\dagger^{\dagger}$ Department of Economics, University Carlos III de Madrid; mdrugov@eco.uc3m.es.

${ }^{\ddagger}$ Department of Economics, University of Oxford; marta.troyamartinez@economics.ox.ac.uk. 
plans and, moreover, the buyer is not sure about her needs, which will only become fully known through the use of the mobile. The seller, who knows the product's features but may not know how they fit with the buyer's needs either (and hence the product quality of match), not only sets a price, but also provides some sales advice to the prospective buyer with the goal of convincing her to buy. In doing so, he can choose to be more or less precise and can also simply lie the buyer by exaggerating the goodness of the product. Sending a misleading sales pitch comes, however, at a cost of customers' complaints that will damage seller's reputation, draw the attention of a consumer association, trigger an action by the competition authority or even result in a litigation.

Some other goods also share these features. For example, consumer electronics, insurance, banking and medical care contracts are all characterized by the fact that neither sellers nor buyers themselves fully know the ex-ante buyers' quality of match with the product, sellers' advice is widely used and buyers learn through experience $^{1}$. Also, these very products systematically top in the list of consumers complaints worldwide which brings about the policy relevance of the problem. For instance, the Federal Trade Commission in the United States has released a report listing top complaints consumers filed with the agency in 2009. ${ }^{2}$ Among the top 15 places it is possible to find health care, internet services, credit cards, advance-fee loans, credit protection, banks and lenders in general and computer equipment and software. Similarly, in the United Kingdom, the Office of Fair Trading has reported (through Consumer Direct, its telephone and online service provider of information on consumer rights) that among the top 10 complaints, it is possible to find mobile phones (service agreements), mobile phones (hardware), telephone services (land line), lap-tops, notebooks and tablet PCs. ${ }^{3}$

We explore the seller's incentives to provide (un)biased and (im)precise advice and the resulting equilibrium communication in the following model. The seller advises the buyer about a certain product trying to increase the buyer's perceived valuation of it. The buyer's valuation for the product is determined by the quality of the match between the product characteristics (unknown to the buyer) and buyer's taste (unknown to the seller). The buyer and the seller share the same prior about quality of the match, that is, none of them has any private information. ${ }^{4}$ The seller

\footnotetext{
${ }^{1}$ In the markets we have in mind, the seller may have private information on how his product compares to competitors but not on how it matches with each buyer's needs. Moreover, the buyer will only learn her actual needs through the use of the product.

${ }^{2}$ See: http://www.ftc.gov/opa/2010/02/2009fraud.shtm (accessed on the 06 December 2011).

${ }^{3}$ See: http://www.oft.gov.uk/shared_oft/general_policy/OFT1267.pdf pp. 21-24 (accessed on the 06 December 2011).

${ }^{4} \mathrm{An}$ alternative interpretation is that the seller is facing the whole demand curve rather than an
} 
gives an advice (i.e., sends a signal) to the buyer about the product. The signal is the sum of the true match quality and an error term that represents frictions in the communication between the seller and the buyer. ${ }^{5}$ Both the match quality and the error term are distributed normally and the seller secretly chooses the mean and publicly the variance of the error term. ${ }^{6}$ We call them "bias" and "noise", respectively.

Upon receiving the signal, the buyer updates her beliefs about the match quality using her conjecture about the bias introduced by the seller (which has to be correct in the equilibrium). This posterior valuation of the product is the surplus generated if trade occurs and that the seller and the buyer share in a fixed proportion. This reflects the popular use of negotiation in selling banking or mobile phone contracts. While the bias unambiguously increases the perceived quality of the product and thus the seller's revenues, the effect of the noise is more subtle and depends on the particular model used. ${ }^{7}$ We adopt a specification where the noise does not affect seller's revenues in the equilibrium, that is, when the buyer correctly anticipates the bias.

Misleading the buyers is costly since eventually, through use, they realize the true quality of the match and complain if the signal they received was much larger. These complaints trigger an action by an authority which might be a consumer protection authority (like Office of Fair Trading), a sectorial regulator (like Financial Services Authority) or the court depending on the product and the country. The authority then investigates the seller surveying a random sample of customers or sending mystery shoppers. Based on this information, it estimates the bias and determines whether there is enough evidence to conclude that the seller has misled the consumer by biasing his signal. More precisely, the authority presumes the innocence of the seller, that is, its null hypothesis is that there has been no bias. ${ }^{8}$ It then tests whether this null hypothesis can be rejected in favor of the alternative hypothesis of a positive bias. In doing so, it uses some given significance level which can be interpreted as a standard of proof. If the seller is found guilty, he has to pay a fine that depends on the estimated bias. A larger bias always increases the costs, i.e., the expected fine. The noise affects the costs through two channels: it decreases the

individual buyer as, for example, in Johnson and Myatt (2006).

${ }^{5}$ The error term can also represent the fact that the true match quality will be learned only through experience.

${ }^{6}$ We also consider the unobservable choice of variance in Section 5.1.

${ }^{7}$ See, for example, Lewis and Sappington (1994) and Johnson and Myatt (2006) where the seller always prefers to provide an extreme (i.e., maximum or minimum) amount of information.

${ }^{8}$ The presumption of innocence is natural in court. When it is a competition authority that punishes the seller the presumption of innocence is explained by the fact that the competition authority may need to defend its position in court if the seller decides to appeal. 
probability that the seller is found guilty but increases the fine when the seller is found guilty. The total effect is U-shaped. ${ }^{9}$

We derive closed form solutions for the equilibrium bias and noise and find that they are complements. When the authority uses a stricter standard of proof or the match quality is more heterogenous, the seller both biases the signal more and makes it more noisy. For example, when buyers heterogeneity is higher, they put a higher weight on the seller's advice and, therefore, the seller has higher incentives to bias it. A higher noise then used since it is complementary to the bias. Introduction of punitive damages makes the seller send a less biased and more precise signal.

We also consider the case of a fixed price of the good. The goal of the seller is then to convince the marginal buyer to buy while in the baseline model it is to increase the posterior of every buyer since everybody buys there. In the special case when the price is equal to the prior mean, we derive closed-form solutions for equilibrium bias and noise and show that they are also complements. Interestingly, a higher buyer's heterogeneity decreases the equilibrium bias and noise, contrary to the baseline mode described above. When the price equals to the mean valuation of the good the only effect of a higher buyer's heterogeneity is to decrease the probability of the buyer with the average valuation, that is, the marginal buyer. Then, the marginal revenues of the bias are smaller and the seller uses it less (and correspondingly he introduces a lower noise). When the price is above (below) the mean valuation, the bias-to-noise ratio is also higher (lower). When the price is above the prior mean, the seller serves relatively high valuation consumers; the noise is relatively low since more information increases the posterior of the marginal high valuation consumer. In the case of the price below the prior mean, the marginal consumer has a relatively low valuation and providing information only decreases her posterior. Therefore, the noise is relatively high.

When two sellers sell related products and buyers can get informed from both of them (although they are locked in and do not choose from whom to buy), there is a disciplining effect. Since each buyer attaches some weight to the other seller's signal, she puts less weight on the signal of her seller. Then, each seller has less incentives to bias his signal and the equilibrium bias and noise go down. Surprisingly, if there are also common shocks in the communication technology (that is, the error terms are correlated), the disciplining effect of the second seller can be reversed. The consumer trusts more her seller's signal since she gets the information about the error term from the second seller and, as a result, the equilibrium bias and noise may increase.

\footnotetext{
${ }^{9}$ In many situations, especially with repeated purchases, reputational concerns also put a limit on the seller's bias. Our model is thus better suited for one-off or infrequent purchases.
} 
We consider a number of other extensions. In the baseline model the noise is observable by both the buyers and the authority. We consider the case of non-observable noise and show that the equilibrium has the same structure and, in particular, equilibrium bias and noise are still complements. In the baseline model the buyers are rational and are not misled in the equilibrium while in reality some consumers do appear naive, at least, to some extent (see examples in Gabaix and Laibson (2006) and Inderst and Ottaviani (2009a) among others). We consider an extension where a proportion of consumers are credulous and blindly follow the seller's advice. We find that the seller then sends a more biased and less precise signal.

\section{$1.1 \quad$ Related literature}

From the theory perspective, this model is related to the literature on career concerns (see Holmström (1999) and Dewatripont, Jewitt, and Tirole (1999)). In a two-period version of these models, the worker's expected second-period performance is his ability since he will not make any effort. The ability is not observed and firms estimate it based on the first-period worker's performance. ${ }^{10}$ The worker then exerts some effort in the first period in order to improve his performance and trick firms into thinking that he has a higher ability, so that they pay him a larger wage. In the equilibrium the firms are not tricked; instead, they anticipate the worker's effort and estimate the worker's ability correctly. Our model is a signal-jamming model as the career concerns models. However, our seller not only jams the signal by manipulating its mean (what we call the "bias"), but also by changing its variance (the "noise") and, hence, its information content.

The choice of variability as a strategic variable has been considered in a large variety of setups. ${ }^{11}$ The general conclusion of these papers is that the players that are at disadvantage tend to choose more risky strategies than those who are in a favorable position, that is, they "gamble for resurrection". In our model the seller's expected revenues are additive in the expected quality and hence his marginal incentives do not depend on it.

Johnson and Myatt (2006) consider how much product relevant information a monopolist would want to provide to its potential customers. Using the fact that

\footnotetext{
${ }^{10}$ The output usually equals to the sum of the effort and the ability. See Dewatripont, Jewitt, and Tirole (1999) for a more general setup.

${ }^{11}$ For instance, Anderson and Cabral (2007) analyze a model of R\&D races where there are two contestants who are allowed to choose the variance of their stochastic R\&D technology. Tsetlin, Gaba, and Winkler (2004) consider the choice of variability of the performance distribution in a multi-round contest. Kräkel, Nieken, and Przemeck (2008) undertake a similar analysis in the auction context.
} 
information about the product rotates the demand curve, they show that the monopolist profits are convex in the information. This generalizes the result of Lewis and Sappington (1994) who showed that the monopolist supplies either maximal or minimal information. In both papers, the choice of the amount of information is costless for the seller, while in our setup this choice is endogenously linked with future fines and we also allow for the possibility of biasing the signal. Furthermore, both papers only consider the case when the amount of information is observable while we also analyze the unobservable case. ${ }^{12}$ A different model is the one of Spiegler (2006) where firms send noisy, unbiased and costless signals of their prices as obfuscation strategies in order to soften competition. In our model, there is no competition, the signal is biased (and noisy) and costly.

The idea that introducing noise might make the bias less costly is also present in $\mathrm{Li}$ (2010) where the sender shares the blame for the wrong message with the noisy and possibly biased intermediary. In Blume and Board (2010) noisy messages are useful in mitigating the conflict between the sender and the receiver in a cheap-talk model.

As we discussed in the beginning, this paper is motivated by the markets where consumers often complain and, therefore, is related to the literature on consumer protection. ${ }^{13}$ Inderst and Ottaviani (2009b), for instance, analyze contract cancellation and product return policies in a market where a more informed seller advises the buyer. Cancellation or return are costly for the seller and, therefore, the advice cannot be too misleading. Since advice in their model takes the form "to buy" or "not to buy", there is no room for the seller to use noise, only bias.

The revenue part of our model can be seen as a cheap-talk model. The interests of the buyer and the seller are opposed since the seller always wants to charge a higher price while the buyer always wants to pay a lower price. Then, there is no information transmission in the equilibrium as is well known starting from the work of Crawford and Sobel (1982). ${ }^{14}$ Then, some exogenous (unmodelled) costs are usually added such as effort costs in career concerns models, refund costs in Inderst and Ottaviani

\footnotetext{
${ }^{12}$ To get an intuition of why observability matters, imagine that the product is bad on average, that is, in the absence of any information the buyer will not buy it (at a given price). To convince the buyer to buy, the seller has to generate a high realisation of the signal and wants the buyer to attach a high weight to this signal in her updating. If the noise is observable, adding noise to the signal will make the buyer attach a lower weight to the signal realization so the optimal strategy is actually to reduce the noise as much as possible (see Johnson and Myatt (2006) for the proof). However, if the noise is not observable, the buyer will not adjust the weight given to the signal to account for the noise and, therefore, the optimal strategy of the seller is to increase the noise of the signal.

${ }^{13}$ See Vickers (2004) and Armstrong (2008) for an overview on this literature.

${ }^{14}$ See, however, Chakraborty and Harbaugh (2010) where information transmission is possible in a multidimensional cheap-talk model despite opposed preferences.
} 
(2009b), or unspecified lying costs in Kartik (2009). Contrary to these papers, in our model the lying costs, which are the expected fine imposed on the seller, are microfounded.

The rest of the paper is organized as follows. Section 2 introduces the model. Section 3 finds the equilibrium and derives comparative statics results. Section 5 contains various extensions and modifications of the baseline model of Section 2 . Section 6 concludes.

\section{The model}

Buyers approach the seller in order to get informed about his product and buy it. The seller does not know their valuations for the product while the buyers do not know the quality of the product and its features. Thus, at the beginning of the interaction, the match quality of the transaction, $\theta$, is unknown both to the seller and the buyer. However, they know that $\theta$ is distributed as $\mathcal{N}\left(\mu, \sigma^{2}\right) .{ }^{15}$ The production costs are normalized to zero.

\subsection{The communication process}

The seller reveals product characteristics and gives advise about the shopping decision to the buyer. In doing so, he can distort the communication strategically. The seller can exaggerate some positive features of the product and he can also be vague about them. More precisely, the seller sends an informative but possibly biased and noisy signal $S$ which takes the following form:

$$
S=\theta+\varepsilon,
$$

where $\varepsilon$ is the distortion introduced by the seller. It is distributed normally, $\varepsilon \rightsquigarrow$ $\mathcal{N}\left(\beta, \eta^{2}\right)$, and both moments are controlled by the seller. ${ }^{16}$ We refer to $\beta$ as bias and to $\eta^{2}$ as noise. The signal is therefore distributed as $\mathcal{N}\left(\mu+\beta, \sigma^{2}+\eta^{2}\right)$; denote its pdf by $g$. The buyer does not observe the bias since she cannot know if a certain feature is exaggerated without actually buying the product and trying it. She does observe

\footnotetext{
${ }^{15}$ As standard in career concerns models, we adopt a setting where the two sides start with the same prior to avoid issues of signalling. See Holmström (1999) and Dewatripont, Jewitt, and Tirole (1999).

${ }^{16}$ The might be some natural noise in the communication process. We discuss this possibility in Section 5.6.
} 
the noise, however, since she can evaluate how precise the seller's explanations are, how much into details he goes, whether there is a trial period, etc. ${ }^{17}$

\subsection{Buyers' valuation and seller's revenues}

The buyer values a better quality of match of the product, $\theta$. For simplicity, the buyer's valuation of the product is linear in the match quality $\theta$ and normalized to be equal to it. Since this quality is only known through use, her valuation at the moment of purchase is the expected match quality given a particular realisation $s$ of the signal.

When the buyer observes a realisation $s$ of the signal and the noise $\eta^{2}$, she updates the expected quality in the standard way using a conjecture about the seller's bias $\widetilde{\beta}:^{18}$

$$
E[\theta \mid s, \eta, \widetilde{\beta}]=\mu+(s-\mu-\widetilde{\beta}) \frac{\sigma^{2}}{\sigma^{2}+\eta^{2}} .
$$

The buyer takes the signal into account with the weight proportional to the prior variance. It can also be written as $\left(\frac{\mu}{\sigma^{2}}+\frac{s-\widetilde{\beta}}{\eta^{2}}\right) /\left(\frac{1}{\sigma^{2}}+\frac{1}{\eta^{2}}\right)$, that is, as the weighted average of the ex-ante quality of match and the ex-post signal realisation (corrected for the bias), where the weights are precisions of the prior and the signal.

Instead of fixing the price before sending the signal, the seller charges the buyer some (fixed) fraction of her valuation which is normalized to 1 . That is, the seller sees the effect that the realisation of his signal, $s$, has had on the buyer and hence, he knows that the buyer is ready to pay up to $E\left[\theta \mid s, \eta^{2}, \widetilde{\beta}\right]$ for the product. This entails some negative payments which can be made negligible by making the average quality $\mu$ high enough. ${ }^{19}$ It also means that the seller always sells to the consumer. This is in the spirit of the career concerns models, where a worker always gets a wage. The only reason it is done both there and in this paper is technical as it allows to integrate over the whole support of the distribution. ${ }^{20}$

Therefore, the seller's revenues are equal to the expected valuation of the buyer:

\footnotetext{
${ }^{17}$ We discuss the case of unobservable noise in Section 5.1.

${ }^{18}$ We assume that the buyer's conjecture does not depend on the observed noise. This assumption is used in the literature, see, for example, Judd and Riordan (1994). Also, this makes the model with unobservable noise (Section 5.1) similar to the model of this section. It would be interesting, though, to investigate other possibilities since different equilibria are then obtained as is shown by Shelegia (2011).

${ }^{19}$ As we will see later, $\mu$ does not affect equilibrium bias and noise (Corollary 1 ).

${ }^{20}$ In a different model, Spiegler (2006) also allows negative prices for technical reasons.
} 


$$
R(\beta, \eta)=\int_{-\infty}^{+\infty} E[\theta \mid s, \eta, \widetilde{\beta}] g(s) d s=\mu+(\beta-\widetilde{\beta}) \frac{\sigma^{2}}{\sigma^{2}+\eta^{2}}
$$

Note that the seller extracts from the buyer the prior expected quality of match $\mu$ plus how much the buyer is misled into thinking that the product is better than it is, adjusted for the weight. In equilibrium, when the buyer's conjecture about the bias used is correct, the seller will only be able to extract the prior expected match quality $\mu$. The revenues decrease with the noise $\eta$ (if $\beta>\widetilde{\beta}$ ) since the buyers pay less attention to the signal, i.e., place a smaller weight on it.

\subsection{Estimated bias and seller's costs}

When the buyers buy the product and start using it, they discover the true match quality. Some buyers, especially those with a large gap between the advice (signal) received from the seller and the true quality, will complain to a public body that we call "authority" throughout the paper. This might be a consumer association, consumer protection agency, a competition authority, an industry regulator or the court, depending on the good or service in question and the country. We assume that this authority can inflict a punishment on the seller. Depending on the nature of the authority, the punishment may be publishing a negative report, ordering to withdraw a certain advertisement, prohibiting a certain commercial practice or imposing a fine on the seller. For the sake of simplicity, we adopt the last meaning and treat the punishment as a monetary fine.

The bias, as a deliberate and conscious way to mislead consumers, is illegal and a fine is imposed on the seller if any positive bias is discovered. In the European Union, the Unfair Commercial Practices Directive 2005/29/EC defines a commercial practice as misleading "...if it contains false information and is therefore untruthful or in any way, including overall presentation, deceives or is likely to deceive the average consumer..." (Article 6). Such practices are more generally called unfair and "...shall be prohibited" (Article 5$).{ }^{21}$

The noise, however, is not punished since it may come from some (unmodelled)

\footnotetext{
${ }^{21}$ In the common law tradition, misrepresentation is a contract law concept which means a false statement of fact made by one party to another party, which has the effect of inducing that party into the contract. For example, under certain circumstances, false statements or promises made by a seller of goods regarding the quality or nature of the product that the seller has may constitute misrepresentation. In English law, misrepresentation is regulated generally by the United Kingdom Misrepresentation Act 1967 and, in the consumer protection area, by Trade Descriptions Act 1968 and Consumer Protection Act 1987.
} 
external shocks in the communication process between the seller and the buyers. The seller may provide information that is vague and open to interpretation, so that the buyers themselves make personal, independent, errors of interpretation. Even if the seller does not bias the signal at all, there will be always some unlucky buyers that will receive a very high signal realisation as compared to their true match quality (unless the communication is absolutely noiseless). For instance, a bad quality of the information at the point of purchase may be due to incompetent sales staff who have trouble giving a clear advice. If these incompetent sales staff are nonetheless objective (i.e., they do not use bias), the buyers will not be misled on average.

The authority conducts an investigation by taking a random sample of size $N$ of buyers in order to estimate the bias introduced by the seller. It can also send "mystery shoppers" to the seller each of them reporting afterwards their experience. In the US and in the UK the competition and consumer protection authorities (FTC and OFT, respectively) routinely commission mystery shopping exercises as part of their market studies. ${ }^{22}$

Each mystery shopper (or buyer) $i=1 . . N$ reports signal $s_{i}$ received from the seller and quality $\theta_{i}$. In Section 5.5 we consider the case when only signals are observed. Having received their reports, the authority uses a statistical test to determine if the seller is guilty of having introduced the bias. ${ }^{23}$ It computes the error terms $\varepsilon_{i}=s_{i}-\theta_{i}$ and estimates the bias used as:

$$
\widehat{\beta}=\frac{1}{N} \sum_{i=1}^{N} \varepsilon_{i}
$$

Since $\varepsilon_{i} \rightsquigarrow \mathcal{N}\left(\beta, \eta^{2}\right)$, this estimator is distributed as $\mathcal{N}\left(\beta, \frac{\eta^{2}}{N}\right)$.

There is the presumption of innocence, that is, by default the seller is assumed not to have introduced any bias, unless enough evidence is provided. In order to determine how convincing the evidence about the use of bias should be the authority

\footnotetext{
${ }^{22}$ See http://www.ftc.gov/os/2009/12/P994511violententertainment.pdf and http://www.oft.gov.uk/shared_oft/business_leaflets/credit_licences/OFT1265.pdf for recent examples (accessed on the 06 December 2011). Other tests are often conducted by courts and consumer bodies. For instance, "copy tests" are used to determine whether an advert is misleading. If enough consumers are misled, the consumer protection authority may order the advert to be withdrawn. The evidence may also come from a class action, see Issacharoff (1999) for a discussion of class actions and consumer protection.

${ }^{23}$ Scholars in law and economics have long been modelling court decision making as probabilistic, see Polinsky and Shavell (2000) for a survey. In legal literature it is also "... now generally accepted that since all the evidence is probabilistic ... evidence should not be excluded merely because its accuracy can be expressed in explicitly probabilistic terms..." (Posner (2004), p. 370). See also Miceli (1990) and Davis (1994) for describing statistical testing in relationship with court decision making.
} 
uses a standard hypothesis test where the null hypothesis of no bias, $H_{0}: \beta=0$, is assessed against the alternative $H_{1}: \beta>0$. The authority constructs the statistics $\frac{\widehat{\beta}}{\eta / \sqrt{N}}$ which, under the null $H_{0}$, is distributed as $\mathcal{N}(0,1)$. Denote $z_{\alpha}$ the threshold such that the seller is found guilty of biasing if and only if $\frac{\widehat{\beta}}{\eta / \sqrt{N}} \geq z_{\alpha}$, where $\alpha$ is the significance level of the test (i.e., the probability of incorrectly rejecting the null hypothesis). ${ }^{24}$ A natural interpretation of $z_{\alpha}$ is the "standard of proof". A higher $z_{\alpha}$ (lower $\alpha$ ) means that it is more difficult to reject the null, and therefore, the authority needs more evidence to be convinced. For instance, if $\alpha$ is $5 \%$, then $z_{\alpha} \approx 1.64$, and if $\alpha$ is $10 \%$, then $z_{\alpha} \approx 1.28$.

If the seller is found guilty, he is imposed a fine which is an increasing function of the estimated bias $\widehat{\beta}$. In this section, we take the fine to be equal to $\widehat{\beta}$ and consider punitive damages $d \widehat{\beta}$ in Section 5.4. The seller's costs are the expected fine:

$$
\begin{aligned}
C(\beta, \eta) & =\frac{\eta}{\sqrt{N}} \int_{z_{\alpha}}^{+\infty} z d H(z) \\
& =\beta\left(1-\Phi\left(z_{\alpha}-\frac{\beta \sqrt{N}}{\eta}\right)\right)+\frac{\eta}{\sqrt{N}} \phi\left(z_{\alpha}-\frac{\beta \sqrt{N}}{\eta}\right)
\end{aligned}
$$

where $z \rightsquigarrow \mathcal{N}\left(\beta \frac{\sqrt{N}}{\eta}, 1\right)$ and $H$ is its cdf (it is the distribution of $\frac{\widehat{\beta}}{\eta / \sqrt{N}}$ ); and $\Phi$ and $\phi$ are cdf and pdf of the standard normal random variable, respectively. The first term in (4b) corresponds to how much the seller pays on average multiplied by the probability of being found guilty. The second term corrects for the selection bias as the truncation selects higher values of $\widehat{\beta}$. The crucial property of this cost function is that it is U-shaped with respect to the noise. A higher $\eta$ makes it less likely for the seller to be found guilty (since the distribution of $z$ shifts to the left, the integral in (4a) decreases), but increases the chances of a really large fine once he is found guilty (this integral is multiplied by a larger number). This is the next lemma. See also Figure 1.

Lemma 1 The seller's costs (4b) are increasing in $\beta$ and U-shaped with respect to $\eta$.

Proof. The first derivative of (4b) with respect to $\beta$ is

$$
\frac{\partial C(\beta, \eta)}{\partial \beta}=1-\Phi\left(z_{\alpha}-\frac{\beta \sqrt{N}}{\eta}\right)+z_{\alpha} \phi\left(z_{\alpha}-\frac{\beta \sqrt{N}}{\eta}\right)
$$

\footnotetext{
${ }^{24}$ By definition, the probability that the standard normal random variable takes a value above $z_{\alpha}$ is equal to $\alpha$.
} 


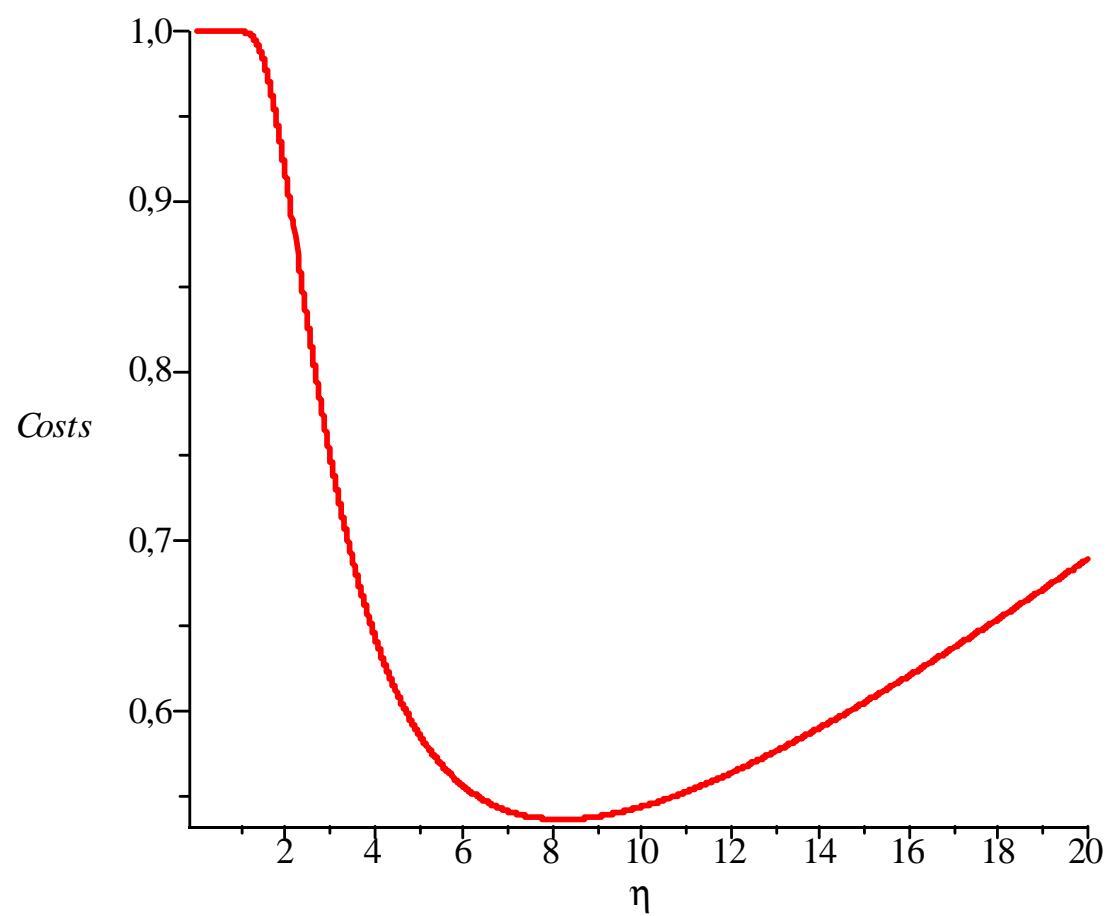

Figure 1: Seller's costs as a function of noise $\eta ; \beta=1, N=25, z_{\alpha}=1.64$.

and it is always positive. The first derivative of (4b) with respect to $\eta$ is

$$
\frac{\partial C(\beta, \eta)}{\partial \eta}=\frac{1}{\sqrt{N}} \phi\left(z_{\alpha}-\frac{\beta \sqrt{N}}{\eta}\right)\left[1-\frac{\beta \sqrt{N}}{\eta} z_{\alpha}\right]
$$

and it is negative for $\eta<\beta \sqrt{N} z_{\alpha}$ and it is positive for $\eta>\beta \sqrt{N} z_{\alpha}$.

\section{Equilibrium}

The seller maximizes his profits which are equal to the revenues (2) minus the costs (4b):

$\Pi(\beta, \eta)=\mu+(\beta-\widetilde{\beta}) \frac{\sigma^{2}}{\sigma^{2}+\eta^{2}}-\beta\left(1-\Phi\left(z_{\alpha}-\frac{\beta \sqrt{N}}{\eta}\right)\right)-\frac{\eta}{\sqrt{N}} \phi\left(z_{\alpha}-\frac{\beta \sqrt{N}}{\eta}\right)$

The equilibrium is a pair $\left(\beta^{*}, \eta^{*}\right)$ that maximizes seller's profits $\Pi(\beta, \eta)$ given buyer's conjecture $\beta^{*}$. Next proposition derives the equilibrium in a closed form. 
Proposition 1 The equilibrium bias and noise are

$$
\begin{aligned}
\beta^{*} & =\frac{\sigma}{\sqrt{N}} \frac{1}{z_{\alpha}} \sqrt{\kappa}, \\
\eta^{*} & =\sigma \sqrt{\kappa},
\end{aligned}
$$

where $\kappa=\frac{\Phi\left(z_{\alpha}-\frac{1}{z_{\alpha}}\right)-z_{\alpha} \phi\left(z_{\alpha}-\frac{1}{z_{\alpha}}\right)}{1-\Phi\left(z_{\alpha}-\frac{1}{z_{\alpha}}\right)+z_{\alpha} \phi\left(z_{\alpha}-\frac{1}{z_{\alpha}}\right)}$.

Proof. See Appendix. ${ }^{25}$

The noise does not affect the revenues in the equilibrium since the buyer is not misled and pays the prior expected match quality $\mu .{ }^{26}$ Thus, for any bias, the noise can be seen as minimizing the costs for that bias. As the cost function is U-shaped with respect to the noise (Lemma 1 ) this yields an interior solution $\frac{\beta \sqrt{N}}{\eta}=\frac{1}{z_{\alpha}} \cdot{ }^{27}$ The bias increases the revenues by changing the signal distribution in the first-order stochastic dominance sense; the marginal effect of the bias on the revenues is the weight of the signal in the buyer's posterior, $\frac{\sigma^{2}}{\sigma^{2}+\eta^{2}}$. The marginal cost of the bias depends only on the ratio $\frac{\beta}{\eta}$ which is pinned down from the cost-minimization with respect to the noise. A closed-form solution can then easily be found.

The comparative statics results are summarized in the next corollary.

Corollary 1 The comparative statics of the equilibrium bias and noise (5a-5b) is the following:

- $\frac{\partial \beta^{*}}{\partial \mu}=\frac{\partial \eta^{*}}{\partial \mu}=0$;

- $\frac{\partial \beta^{*}}{\partial \sigma^{2}}, \frac{\partial \eta^{*}}{\partial \sigma^{2}}>0$;

- $\frac{\partial \beta^{*}}{\partial z_{\alpha}}, \frac{\partial \eta^{*}}{\partial z_{\alpha}}>0$;

- $\frac{\partial \beta^{*}}{\partial N}<0$ and $\frac{\partial \eta^{*}}{\partial N}=0$.

Given that a change in any parameter does not affect the equilibrium bias and noise in opposite directions, we say that they are complements in the equilibrium.

The average quality $\mu$ does not affect the bias and the noise. Since the seller sells for any realisation of the signal and the buyer's valuation is linear in the quality,

\footnotetext{
${ }^{25}$ We need to assume that $z_{\alpha}<2.436$, that is, $\alpha>0.75 \%$, for the second-order conditions to hold.

${ }^{26} \mathrm{~A}$ higher noise increases the revenues if $\beta<\widetilde{\beta}$ and decreases them if $\beta>\widetilde{\beta}$ since it decreases the signal weight in the buyer's posterior.

${ }^{27}$ The cost function is homogeneous of degree 1 in $\beta$ and $\eta$; therefore, the marginal cost functions are homogeneous of degree 0 in $\beta$ and $\eta$, that is, they depend only on the ratio $\frac{\beta}{\eta}$.
} 
the seller's expected revenues are additive in $\mu$. His marginal incentives then do not depend on $\mu$.

When the prior is less precise, that is, $\sigma^{2}$ is larger, it becomes more profitable to mislead the consumer because more attention is paid to the signal. Hence, the bias is used more. The costs are unaffected by $\sigma^{2}$ per se. However, as the bias increases, a higher noise should be used to bring the "standardized" bias $\frac{\beta \sqrt{N}}{\eta}$ down to its optimal level $\frac{1}{z_{\alpha}}$. Also, the noise is proportional to $\sigma^{2}$ since it is the ratio of the two that determines the weights in the buyer's posterior (1).

The variance of the prior $\sigma^{2}$, which is the buyers' heterogeneity, can be thought of as affected by the seller at an earlier stage through the product design. In Johnson and Myatt (2006) the relationship between the buyers' heterogeneity and the informativeness of the signal is the opposite: the benefits of giving precise information are higher if the buyers differ largely in their tastes and, therefore, more idiosyncratic products are complemented by detailed advertising and marketing activities. Therefore, if litigation is possible and the revenues are not affected by the noise, we should expect more heterogeneity in the product design to come together with larger bias and noise. Note that heterogeneity in the product design is a salient feature of the markets we described in the introduction.

A stricter (i.e., higher) standard of proof $z_{\alpha}$ means that it is more difficult to convict the seller, so the bias becomes cheaper. However, it also increases the noise that minimizes the costs for any given bias. Indeed, since there are less chances to convict the seller, the selection bias problem mentioned in Section 2.3 becomes less of an issue. The larger noise decreases the marginal revenues of the bias because the buyers pay less attention to the advice. Overall, we find that this decrease in the marginal revenues is always dominated by the decrease in the marginal cost, and as a result, the bias always increases following an increase in $z_{\alpha}$.

When the authority increases the sample size $N$, the seller should counteract this increase in the precision of the bias estimation by increasing the noise (i.e., the cost minimizing noise shifts to the right). A larger noise decreases the marginal revenues of the bias (through a lower weight on the advice in the buyer's updating) and the bias is used less as a result. Since the seller biases less his advice, he also needs less noise to "hide" his misleading practice. It turns out that the noise decreases by enough to compensate the original increase which leads to the quite surprising result that the equilibrium noise does not depend on $N$.

Let us conclude this section with the following observation. In our model, setting a sufficiently high sample size $N$ or a sufficiently low standard of proof $z_{\alpha}$ (or a sufficiently high punitive damages $d$ that we investigate in Section 5.4) drives equilibrium 
noise and bias to zero. We would not, however, overestimate practical implications of this result for two reasons. First, this might not be the case if there is some natural and irreducible noise in communication between the buyer and the seller (see Section 5.6), as there will be then some lower bound on the equilibrium noise. Second, a richer model (which is beyond the scope of this paper and is an interesting topic for future work) is needed for a study of the optimal policy. Such a model will have physical costs of sending $N$ mystery shoppers and welfare costs of punishing innocent sellers. Then, allowing for positive noise and bias in the equilibrium might be optimal.

\section{Fixed price}

In many cases the price of a product is fixed and the buyer decides whether to buy it or not at that given price. It is an interesting question (for future work) how the pricing strategy interacts with the choice of bias and noise. Assume for now that the price, $p$, is exogenously fixed at a pre-advice stage. ${ }^{28}$ The buyer decides to buy if her posterior valuation of the product (1) is higher than $p$. The seller's revenues are then

$$
R(\beta, \eta)=p \operatorname{Pr}\left\{\frac{(s-\widetilde{\beta}) \sigma^{2}+\mu \eta^{2}}{\sigma^{2}+\eta^{2}} \geq p\right\}=p\left(1-G\left(p+\widetilde{\beta}+(p-\mu) \frac{\eta^{2}}{\sigma^{2}}\right)\right)
$$

and the seller's costs are unchanged.

Consider first the case where the price is exogenously fixed at the mean level $\mu$.

Proposition 2 When $p=\mu>0$, the equilibrium bias and noise are:

$$
\begin{aligned}
\beta_{p}^{*} & =\frac{\sqrt{\max \left\{\frac{\mu^{2}}{2 \pi \lambda^{2}}-\sigma^{2}, 0\right\}}}{z_{\alpha} \sqrt{N}} \\
\eta_{p}^{*} & =\sqrt{\max \left\{\frac{\mu^{2}}{2 \pi \lambda^{2}}-\sigma^{2}, 0\right\}}
\end{aligned}
$$

where $\lambda=1-\Phi\left(z_{\alpha}-\frac{1}{z_{\alpha}}\right)+z_{\alpha} \phi\left(z_{\alpha}-\frac{1}{z_{\alpha}}\right) \cdot{ }^{29}$

Proof. See Appendix. ${ }^{30}$

\footnotetext{
${ }^{28}$ This scenario also corresponds to the case where the price is fixed by the marketing division that operates independently from the selling division.

${ }^{29}$ Then $\kappa$ of Proposition 1 can be written as $\kappa=\frac{1-\lambda}{\lambda}$.

${ }^{30} \mathrm{~A}$ sufficient (but not necessary) condition for the second order conditions to hold is $z_{\alpha} \geq 1.09$ (i.e., $\alpha \leq 13.79 \%$ ).
} 
The seller is trying to convince the marginal buyer. As the noise is observable, it changes the identity of the marginal buyer. In the equilibrium, however, the noise does not affect the seller's revenues since the buyer is not misled. Thus, as in the baseline model of Section 2, the noise can be seen as minimizing the costs for any given bias which pins down the bias-to-noise ratio, $\frac{\beta \sqrt{N}}{\eta}=\frac{1}{z_{\alpha}}$. The effect of the bias is to sell to the marginal buyer, i.e., to the one who receives signal $s=\bar{s}$. In the equilibrium the marginal buyer is the one who has the mean valuation $\mu$ and, therefore the marginal effect of bias on the revenues is $\mu$ times the probability of the mean valuation. Since the latter depends on the noise while the marginal cost of the bias depends only on the ratio $\frac{\beta}{\eta}$, a closed-form solution can then easily be found.

The comparative statics results are summarized in the next corollary.

Corollary 2 The comparative statics of the equilibrium bias and noise (6a-6b) is the following:

- $\frac{\partial \beta_{p}^{*}}{\partial \mu}, \frac{\partial \eta_{p}^{*}}{\partial \mu}>0$;

- $\frac{\partial \beta_{p}^{*}}{\partial \sigma^{2}}, \frac{\partial \eta_{p}^{*}}{\partial \sigma^{2}}<0$;

- $\frac{\partial \beta_{p}^{*}}{\partial z_{\alpha}}, \frac{\partial \eta_{p}^{*}}{\partial z_{\alpha}}>0,31$

- $\frac{\partial \beta_{p}^{*}}{\partial N}<0$ and $\frac{\partial \eta_{p}^{*}}{\partial N}=0$.

Proof. See Appendix.

Given that a change in any parameter does not affect the equilibrium bias and noise in opposite directions, we say that they are still complements in the equilibrium.

The average quality now affects the bias and the noise unlike the baseline model of Section 2. A higher $\mu$ increases both the posterior expected quality and the price, so the probability of selling remains unchanged. However, an increase in $\mu$ (through the increase in $p$ ) also increases the revenue per unit sold. The increase in the profitability of the sale induces the seller to increase the bias, as this increases the probability of selling. Following the increase in the bias, the noise increases too to minimize the costs for this higher bias.

The comparative statics with respect to the variance of the prior, $\sigma^{2}$, have the opposite sign than in Section 3 (see Corollary 1). The intuition is the following. While $\sigma^{2}$ still enters the buyer's posterior, it does not affect her decision to buy.

\footnotetext{
${ }^{31} \mathrm{~A}$ sufficient (but not necessary) condition for $\frac{\partial \beta_{p}^{*}}{\partial z_{\alpha}}>0$ is $z_{\alpha} \geq 1.29$ (i.e., $\alpha \leq 9.85 \%$ ). See the proof for the exact condition.
} 
Indeed, since the price equals to the mean valuation, the buyer buys if the signal exceeds the mean (after debiasing) and does not buy otherwise. Thus, the only effect of a higher $\sigma^{2}$ is to decrease the probability of the marginal buyer, that is, the buyer with the average valuation $\mu$. Then, the marginal revenues of the bias are smaller and the seller uses it less (and correspondingly he introduces a lower noise).

When the standard of proof, $z_{\alpha}$, increases, the seller can afford to use a larger noise as the probability of ending up convicted and paying a large fine decreases. A stricter standard of proof, also decreases the marginal cost of biasing the advice. However, as in the comparative statics of Section 3, the larger noise also decreases the marginal revenue of the bias although through a different mechanism. As with $\sigma^{2}$, a larger noise decreases the probability of the marginal consumer and hence makes biasing the advice less profitable. However, if $z_{\alpha}$ is large enough, the decrease of the marginal revenues is smaller than the one of marginal costs and the bias increases.

The intuition for the comparative statics of the sample size $N$ is the same as in Section 3, with the difference that the decrease in the marginal revenues of bias following an increase in the noise is due to a decrease in the probability of the marginal consumer (and not to a decrease in the attention paid to the advice).

Finally, it is easy to check that if the ratio $\frac{\mu^{2}}{2 \pi \sigma^{2}}$ is large enough (i.e., $\frac{\mu^{2}}{2 \pi \sigma^{2}}>\lambda$ ), then $\beta_{p}^{*}>\beta^{*}$ and $\eta_{p}^{*}>\eta^{*}$. This is because, as shown in Corollary 2, a larger $\mu$ and/or a smaller $\sigma^{2}$ increases the marginal benefit of biasing the advice.

Consider now the case $p \neq \mu$. While we cannot obtain closed-form solutions we still can shed light on the bias-to-noise ratio in the equilibrium.

Proposition 3 When $p>(<) \mu$, then $\frac{\beta_{p}^{*}}{\eta_{p}^{*}}>(<) \frac{\beta^{*}}{\eta^{*}}$, where $\beta^{*}$ and $\eta^{*}$ are defined in (6a) and (6b).

\section{Proof. See Appendix.}

The only difference with the previous setup where $p=\mu$ is that now the noise has two effects on the marginal revenues: like the bias, it affects the decision of the marginal consumer (in the direction that depends on how the marginal consumer is related to the mean of the signal) but, since the noise is observable, it also changes the identity of the marginal consumer. In the equilibrium, a higher noise brings the buyer's posterior closer to the mean which decreases the seller's revenues when $p>\mu$ since the marginal consumer has the valuation above the mean and increases them when $p<\mu$ since the marginal consumer has the valuation below the mean. As a result, when $p>\mu$ the bias-to-noise ratio will be larger than the one in Section 3 and smaller when $p<\mu$. Actually, the analysis of the effects of the noise on 
the seller's revenues becomes essentially the one of Johnson and Myatt (2006). The case $p>\mu$ corresponds to their "niche market", where the seller only serves the high valuation consumers and provides them with a lot of information, and the case $p<\mu$ corresponds to their "mass market" where the seller serves most consumers and gives them few information. However, contrary to their setup where noise is costless, in our model the noise also affects the seller's costs and, therefore, an extreme noise is not optimal.

\section{$5 \quad$ Extensions}

\subsection{Unobservable noise}

In some cases it is more reasonable to assume that the noise chosen by the seller is not observed by the buyer. For instance, the seller can increase the noise by failing to explain hidden costs that the buyer may incur just once he uses the service or some features that the buyer did not think about or expected to use significantly (such as a bank overdraft). The unobserved noise can also come from the small script that is typically attached to this type of contracts. These clauses are usually not read in the moment of the purchase and they can be more or less precise. In all these examples, the buyer cannot assess at the moment of receiving the signal how precise it is.

The buyer updates her beliefs in a way similar to (1):

$$
E[\theta \mid s, \widetilde{\eta}, \widetilde{\beta}]=\mu+(s-\mu-\widetilde{\beta}) \frac{\sigma^{2}}{\sigma^{2}+\widetilde{\eta}^{2}}
$$

where $\widetilde{\eta}$ is the buyer's conjecture about the noise used by the seller (that has to be correct in the equilibrium). The seller's revenues are equal to the expected valuation that the seller extract from the buyers:

$$
R(\beta, \eta)=\int_{-\infty}^{+\infty} E[\theta \mid s, \widetilde{\eta}, \widetilde{\beta}] g(s) d s=\mu+(\beta-\widetilde{\beta}) \frac{\sigma^{2}}{\sigma^{2}+\widetilde{\eta}^{2}}
$$

Note that the seller extracts from the buyer the expected quality of match plus how much the buyer is misled into thinking that the product is good. The only difference with (2) is that the buyer uses her conjecture about the noise $\widetilde{\eta}$ since the noise is not observable. The noise $\eta$ does not affect the revenues then. In equilibrium, when the buyer's conjecture about the bias used is correct, the seller extracts the expected match quality $\mu$ as before. 
If buyers do not observe the noise, the authority does not observe it either and has to estimate it as

$$
\widehat{\eta^{2}}=\frac{1}{N-1} \sum_{i=1}^{N}\left(\varepsilon_{i}-\widehat{\beta}\right)^{2}
$$

where $\widehat{\beta}$ is the estimated bias (3). This estimator is distributed as $\widehat{\eta^{2}} \rightsquigarrow \chi^{2}(N-1)$. The authority constructs statistics $t=\frac{\widehat{\beta}}{\widehat{\eta} / \sqrt{N}}$ which, under the null hypothesis of seller's innocence $\beta=0$ has $t$-distribution with $N-1$ degrees of freedom. The authority rejects $H_{0}$ if $\frac{\widehat{\beta}}{\widehat{\eta} / \sqrt{N}} \geq t_{\alpha}$, where $\alpha$ is the significance level of the test. ${ }^{32}$ However, for a given bias $\beta$, the true distribution of $\frac{\widehat{\beta}}{\hat{\eta} / \sqrt{N}}$ is non-central $t$-distribution with $N-1$ degrees of freedom and non-centrality parameter $n c p=\beta \frac{\sqrt{N}}{\eta}$ (denote its cdf as $\left.T_{N-1}^{n c p}\right) .{ }^{33}$ The seller's costs are

$$
C(\beta, \eta)=\frac{\eta}{\sqrt{N}} \int_{t_{\alpha}}^{+\infty} t d T_{N-1}^{n c p}(t)
$$

The only difference with costs $(4 \mathrm{a})$ is that the normal distribution there is now replaced with non-central $t$-distribution. A higher $\beta$ increases ncp and, therefore, the distribution $T_{N-1}^{n c p}$ moves to the right and the integral in (7) goes up. A higher $\eta$ has two effects, as before: it makes it less likely for the seller to be found guilty (the integral in (7) decreases since $n c p$ is lower), but increases the chances of a really large fine once he is found guilty (the integral in (7) is multiplied by a higher number). It is easily shown that costs $(7)$ are decreasing at $\eta=0$ and increasing at $\eta \rightarrow+\infty$ and, hence, minimized for some interior $\eta$.

The equilibrium then has a similar structure to the one in Section 3 (in particular, equilibrium bias and noise are complements) and is found in a similar way. Now, in the equilibrium $\beta=\widetilde{\beta}$ and $\eta=\widetilde{\eta}$. The first-order conditions of the seller's profit maximization in the equilibrium are

$$
\begin{aligned}
& \frac{\partial \Pi(\beta, \eta)}{\partial \beta}=\frac{\sigma^{2}}{\sigma^{2}+\eta^{2}}-\frac{\partial}{\partial n c p} \int_{t_{\alpha}}^{+\infty} t d T_{N-1}^{n c p}(t)=0 \\
& \frac{\partial \Pi(\beta, \eta)}{\partial \eta}=-\frac{1}{\sqrt{N}} \int_{t_{\alpha}}^{+\infty} t d T_{N-1}^{n c p}(t)+\frac{\beta}{\eta} \frac{\partial}{\partial n c p} \int_{t_{\alpha}}^{+\infty} t d T_{N-1}^{n c p}(t)=0
\end{aligned}
$$

\footnotetext{
${ }^{32}$ By definition, the probability that a random variable following $t$-distribution with $N-1$ degrees of freedom takes a value above $t_{\alpha}$ is equal to $\alpha$.

${ }^{33}$ Indeed,

$\operatorname{Pr}\left\{\frac{\widehat{\beta}}{\widehat{\eta} / \sqrt{N}} \geq t_{\alpha}\right\}=\operatorname{Pr}\left\{\frac{\frac{\widehat{\beta}-\beta}{\eta / \sqrt{N}}+\frac{\beta}{\eta / \sqrt{N}}}{\sqrt{\frac{N-1}{N-1}} \frac{\eta}{\eta}} \geq t_{\alpha}\right\}=\operatorname{Pr}\left\{\frac{\mathcal{N}(0,1)+\frac{\beta}{\eta / \sqrt{N}}}{\sqrt{\frac{\chi_{N-1}^{2}}{N-1}}} \geq t_{\alpha}\right\}=1-T_{N-1}^{n c p}\left(t_{\alpha}\right)$
}

where the last equality is the definition of the non-central $t$-distribution. 
Condition (8b) depends only on $n c p=\beta \frac{\sqrt{N}}{\eta}$ (after multiplying it by $\sqrt{N}$ ) and, therefore, in the equilibrium the ratio $\frac{\beta}{\eta}$ is chosen to minimize the costs with respect to the noise. Then, the noise is found from (8a), in which the second term, the equilibrium marginal costs of bias $\frac{\partial}{\partial n c p} \int_{t_{\alpha}}^{+\infty} t d T_{N-1}^{n c p}(t)$, does not depend on bias and noise.

With observable noise, $\beta \frac{\sqrt{N}}{\eta}$ is also chosen to minimize the costs. Then, $\eta$ is found from $\frac{\partial \Pi(\beta, \eta)}{\partial \beta}=0$. With unobservable noise, it is the buyer's conjecture $\widetilde{\eta}$ that makes the condition $\frac{\partial \Pi(\beta, \eta)}{\partial \beta}=0$ satisfied. Since the equilibrium $\widetilde{\eta}$ is equal to $\eta$, all the difference comes from the fact that the authority statistics has normal distribution in the observable case and non-central $t$-distribution here. It also means that, when $N$ increases and costs $(7)$ converge to the costs in the baseline case (4a) since the non-observability of the noise matters less and less for the authority's estimation, the equilibrium then also converges to the equilibrium in the baseline case (5a-5b).

Finally, when the noise is unobserved, there is no off-equilibrium events. The buyer observes only a signal realization and any realization is compatible with any choice of bias and noise by the seller. The fact that the equilibrium when the noise is unobservable looks similar to the case when the noise is observable is one of the main reasons why we focused on the constant conjecture $\widetilde{\beta}(\eta)$ in the baseline model (see fn. 18).

\subsection{Informational externalities between sellers}

Suppose that, while still buying from a given seller, the buyer also observes a signal from another seller. For example, the buyer may want to buy only a certain brand of a mobile phone, say, for aesthetic or compatibility reasons, but she can learn about some new technical features from the mobile phones of other brands as well. ${ }^{34}$ Sellers cannot distinguish between buyers who buy from them and those who only get informed.

There are two sellers, seller 1 and seller 2, each sending a signal with their respective bias and noise. The buyer has the match quality $\theta_{1} \rightsquigarrow \mathcal{N}\left(\mu_{1}, \sigma_{1}^{2}\right)$ with seller 1 and $\theta_{2} \rightsquigarrow \mathcal{N}\left(\mu_{2}, \sigma_{2}^{2}\right)$ with seller 2 . The correlation between $\theta_{1}$ and $\theta_{2}$ is $\rho_{\theta}$ and the correlation between distortions $\varepsilon_{1}$ and $\varepsilon_{2}$ is $\rho_{\varepsilon}$. The former describes how similar the two products are. Somebody may be determined to buy an iPhone but knows that many functional features are similar in the phones of other brands. The latter reflects some random changes in the buyer's mood or attitude towards. A buyer may

\footnotetext{
${ }^{34}$ See Ivanov (2011) for a persuasion model (in which the bias cannot be used) where the buyer can choose from which seller to buy.
} 
have a bad moment and she would then be critical to any information she receives from the sellers.

Seller $i$ 's signal $s_{i}$ is distributed as $\mathcal{N}\left(\mu_{i}+\beta_{i}, \sigma_{i}^{2}+\eta_{i}^{2}\right)$. Having observed signals from both sellers, $s_{1}$ and $s_{2}$, the buyer who buys from seller 1 computes her expected match quality in the following way (see Appendix for the derivation):

$$
\begin{aligned}
E\left[\theta_{1} \mid s_{1}, s_{2}, \eta_{1}, \eta_{2}, \widetilde{\beta}_{1}, \widetilde{\beta}_{2}\right]=\mu_{1} & +\frac{1+\frac{\eta_{2}^{2}}{\sigma_{2}^{2}}-\rho_{\theta}\left(\rho_{\theta}+\rho_{\varepsilon} \frac{\eta_{1} \eta_{2}}{\sigma_{1} \sigma_{2}}\right)}{\left(1+\frac{\eta_{2}^{2}}{\sigma_{2}^{2}}\right)\left(1+\frac{\eta_{1}^{2}}{\sigma_{1}^{2}}\right)-\left(\rho_{\theta}+\rho_{\varepsilon} \frac{\eta_{1} \eta_{2}}{\sigma_{1} \sigma_{2}}\right)^{2}}\left(s_{1}-\mu_{1}-\widetilde{\beta}_{1}\right) \\
& +\frac{\rho_{\theta} \frac{\eta_{1}^{2}}{\sigma_{1} \sigma_{2}}-\rho_{\varepsilon} \frac{\eta_{1} \eta_{2}}{\sigma_{2}^{2}}}{\left(1+\frac{\eta_{2}^{2}}{\sigma_{2}^{2}}\right)\left(1+\frac{\eta_{1}^{2}}{\sigma_{1}^{2}}\right)-\left(\rho_{\theta}+\rho_{\varepsilon} \frac{\eta_{1} \eta_{2}}{\sigma_{1} \sigma_{2}}\right)^{2}}\left(s_{2}-\mu_{2}-\widetilde{\beta}_{2}\right) .
\end{aligned}
$$

The two sellers move simultaneously. In the equilibrium, when deciding on the bias and noise, each of them knows the bias and noise of the other but not the realisation of the other seller's signal. Also, the buyer has correct conjectures about the biases of both sellers. If seller 1 decides to deviate from the equilibrium to some bias $\beta_{1}$ (when seller 2 does not) his expected revenues are

$$
\begin{aligned}
R_{1}\left(\beta_{1}, \eta_{1}\right) & =\int_{-\infty}^{+\infty} \int_{-\infty}^{+\infty} E\left[\theta_{1} \mid s_{1}, s_{2}, \eta_{1}, \eta_{2}, \widetilde{\beta}_{1}, \widetilde{\beta}_{2}\right] d G\left(s_{1}\right) d G\left(s_{2}\right) \\
& =\mu_{1}+\frac{1+\frac{\eta_{2}^{2}}{\sigma_{2}^{2}}-\rho_{\theta}\left(\rho_{\theta}+\rho_{\varepsilon} \frac{\eta_{1} \eta_{2}}{\sigma_{1} \sigma_{2}}\right)}{\left(1+\frac{\eta_{2}^{2}}{\sigma_{2}^{2}}\right)\left(1+\frac{\eta_{1}^{2}}{\sigma_{1}^{2}}\right)-\left(\rho_{\theta}+\rho_{\varepsilon} \frac{\eta_{1} \eta_{2}}{\sigma_{1} \sigma_{2}}\right)^{2}}\left(\beta_{1}-\widetilde{\beta}_{1}\right) .
\end{aligned}
$$

The costs are still given by $(4 \mathrm{~b}) .{ }^{35}$

Proposition 4 With informational externalities between the two sellers, when correlation is imperfect, $\left|\rho_{\theta}\right|<1$ and $\left|\rho_{\varepsilon}\right|<1$, there is a unique symmetric (pure-strategy) equilibrium

$$
\begin{aligned}
& \beta_{i}^{e}=\frac{1}{z_{\alpha}} \frac{\eta_{i}^{e}}{\sqrt{N}}, \\
& \eta_{i}^{e}=\frac{\sigma_{i}}{\sqrt{2}} \sqrt{(\kappa-1) \frac{1-\rho_{\theta} \rho_{\varepsilon}}{1-\rho_{\varepsilon}^{2}}+\sqrt{(\kappa-1)^{2}\left(\frac{1-\rho_{\theta} \rho_{\varepsilon}}{1-\rho_{\varepsilon}^{2}}\right)^{2}+4 \kappa \frac{1-\rho_{\theta}^{2}}{1-\rho_{\varepsilon}^{2}}}}
\end{aligned}
$$

where $\kappa$ is defined in Proposition 1.

\footnotetext{
${ }^{35}$ Despite the correlation between $\varepsilon_{1}$ and $\varepsilon_{2}$, the estimator of the bias is still the sample average. See Casella and Berger (2002), p. 358, ex. 7.18.
} 
If the match qualities are perfectly correlated, $\left|\rho_{\theta}\right|= \pm 1$, there is always equilibrium $\beta_{1}^{e}=\beta_{2}^{e}=\eta_{1}^{e}=\eta_{2}^{e}=0$. If and only if $\kappa>1$, there is another equilibrium ${ }^{36}$

$$
\begin{aligned}
\beta_{i}^{e} & =\frac{1}{z_{\alpha}} \frac{\eta_{i}^{e}}{\sqrt{N}} \\
\eta_{i}^{e} & =\sigma_{i} \sqrt{\frac{\kappa-1}{1 \pm \rho_{\varepsilon}}} .
\end{aligned}
$$

If the shocks are perfectly correlated, $\left|\rho_{\varepsilon}\right|= \pm 1$, if and only if $\kappa<1$, there is a unique symmetric (pure-strategy) equilibrium

$$
\begin{aligned}
\beta_{i}^{e} & =\frac{1}{z_{\alpha}} \frac{\eta_{i}^{e}}{\sqrt{N}} \\
\eta_{i}^{e} & =\sigma_{i} \sqrt{\frac{\kappa}{1-\kappa}\left(1 \pm \rho_{\theta}\right)}
\end{aligned}
$$

Proof. See Appendix.

The effects of the correlations $\rho_{\theta}$ and $\rho_{\varepsilon}$ are sometimes ambiguous when they are both quite high. If $\rho_{\varepsilon}$ is close to zero, then the effect of $\rho_{\theta}$ is to decrease the equilibrium value of bias and noise. This comes from the fact that when $\rho_{\theta}$ increases, the buyer puts a lower weight on the signal of her seller and a higher one on the signal of the other seller, as can be seen from (9). Then, each seller has less incentives to bias his signal and, correspondingly, he introduces less noise.

When $\rho_{\theta}$ is close to zero, an increase in $\rho_{\varepsilon}$ has an opposite effect on the buyer's posterior. Indeed, the buyer trusts the signal from her seller more since she knows something about the realization of the error term through the correlation between $\varepsilon_{1}$ and $\varepsilon_{2}$. Then, the seller has more incentives to bias his signal and add noise. It can be easily checked that $\beta_{i}^{e}$ and $\eta_{i}^{e}$ in (11) are higher than $\beta^{*}$ and $\eta^{*}$ in $(5 \mathrm{a}-5 \mathrm{~b})$ for $\rho_{\theta}=0$ and $\rho_{\varepsilon} \neq 0$. Thus, the presence of another seller who sells an unrelated good but with correlated shocks in the communication process decreases welfare. The seller cannot commit not to bias his signal more and, while the consumers are not misled in the equilibrium, he ends up paying a higher fine. ${ }^{37}$

When the match quality of one seller is perfectly correlated with the one of the other, $\left|\rho_{\theta}\right|= \pm 1$, the situation is slightly different. If one of the sellers does not use any noise, he perfectly reveals his match quality and, therefore, the match quality of the other seller. The latter then cannot mislead the buyer and chooses zero bias and

\footnotetext{
${ }^{36} \mathrm{~A}$ higher $\kappa$ means a higher standard of proof $z_{\alpha}$ (and a lower $\alpha$ ); $\kappa=1$ corresponds to $z_{\alpha} \approx 1.70$ (and $\alpha \approx 4.5 \%$ ).

${ }^{37}$ See Meyer and Vickers (1997) for a dynamic model of implicit incentives where informational externalities between two agents also may reduce welfare.
} 
noise. There might be another equilibrium where both sellers do use some bias and noise and which is the limit of the equilibrium with imperfect correlation (11).

\subsection{Credulous buyers}

In the baseline model, the buyers are rational and anticipate correctly the bias introduced by the seller. Then, the bias (and the noise) do not harm the buyers in the equilibrium: each of them pays on average $\mu$ which is the average match quality.

However, there is extensive empirical evidence about the existence of credulous buyers. For instance, brokers are usually paid by the consumers but also receive compensation from the lenders - which influence the broker to offer the consumer loan terms or products that are not in the consumer's interest. However, according to the FTC (2008), "many consumers purportedly view mortgage brokers as trusted advisors who shop for the best loan for the consumer" (p. 16). See other examples in Gabaix and Laibson (2006) and Inderst and Ottaviani (2009a) among others.

In this Section, we let some buyers to be credulous and blindly believe the seller's signal. More precisely, a share of credulous buyers $c$ does not understand that the seller might send a biased and imprecise signal. Thus, these consumers blindly follow the signal.

The seller's expected revenues are then

$$
R(\beta, \eta)=\mu+(1-c)(\beta-\widetilde{\beta}) \frac{\sigma^{2}}{\sigma^{2}+\eta^{2}}+c \beta
$$

Proposition 5 When there is share c of credulous consumers, the equilibrium bias and noise are

$$
\begin{aligned}
\beta_{c}^{*} & =\frac{\sigma}{\sqrt{N}} \frac{1}{z_{\alpha}} \sqrt{\kappa_{c}}, \\
\eta_{c}^{*} & =\sigma \sqrt{\kappa_{c}}
\end{aligned}
$$

where $\kappa_{c}=\frac{\Phi\left(z_{\alpha}-\frac{1}{z_{\alpha}}\right)-z_{\alpha} \phi\left(z_{\alpha}-\frac{1}{z_{\alpha}}\right)}{1-\Phi\left(z_{\alpha}-\frac{1}{z_{\alpha}}\right)+z_{\alpha} \phi\left(z_{\alpha}-\frac{1}{z_{\alpha}}\right)-c} \cdot 38$

They both increase with $c$.

Proof. Similar to the proof of Proposition 1.

\footnotetext{
${ }^{38}$ If $1-\Phi\left(z_{\alpha}-\frac{1}{z_{\alpha}}\right)+z_{\alpha} \phi\left(z_{\alpha}-\frac{1}{z_{\alpha}}\right)-c \leq 0$, the seller's marginal costs in the equilibrium are smaller then his marginal revenues and, thus, he introduces an infinite bias.
} 
As expected, equilibrium bias increases with the share of credulous consumers since marginal returns to bias are higher. The equilibrium noise also increases since it is complementary to the bias.

\subsection{Punitive damages}

Punitive (or exemplary) damages are used often in common law countries. In the US, both the frequency and the magnitude of punitive damages verdicts has increased dramatically in recent years (Sunstein, Hastie, Payne, Schkade, and Viscusi (2002)). We can model them by assuming that the seller is fined by the amount $d \widehat{\beta}$ when he is found guilty, $d \geq 1 .^{39}$ The cost function (4b) is then multiplied by $d$. Following the same steps as in the baseline model, we obtain the equilibrium bias and noise.

Proposition 6 When there are punitive damages $d$, the equilibrium bias and noise are

$$
\begin{aligned}
\beta_{d}^{*} & =\frac{\sigma}{\sqrt{N}} \frac{1}{z_{\alpha}} \sqrt{\max \left\{\kappa_{d}, 0\right\}} \\
\eta_{d}^{*} & =\sigma \sqrt{\max \left\{\kappa_{d}, 0\right\}}
\end{aligned}
$$

where $\kappa_{d}=\frac{1-d\left[1-\Phi\left(z_{\alpha}-\frac{1}{z_{\alpha}}\right)+z_{\alpha} \phi\left(z_{\alpha}-\frac{1}{z_{\alpha}}\right)\right]}{d\left[1-\Phi\left(z_{\alpha}-\frac{1}{z_{\alpha}}\right)+z_{\alpha} \phi\left(z_{\alpha}-\frac{1}{z_{\alpha}}\right)\right]}$.

They both decrease with $d$.

Proof. Similar to the proof of Proposition 1.

When $d=1$, we obtain our baseline case results (5a-5b). When the punishment becomes more severe, that is, $d$ increases, there is less bias and less noise. If $d$ is high enough, the seller is completely deterred from misleading the buyers.

\subsection{Only signals are observed ${ }^{40}$}

If it takes a considerable amount of time to learn the quality of the match or there is a lot of subjectivity involved, then, when the authority sends mystery shoppers, they observe only signals $s_{i}, i=1, \ldots, N$. The estimated bias is

\footnotetext{
${ }^{39} \mathrm{We}$ abstract from the fact that in practice it is not very clear how the amount of punitive damages awarded depends on the harm made. Sunstein, Hastie, Payne, Schkade, and Viscusi (2002) say that there "...is inability to explain ... various punitive damages verdicts on a rational basis" (p. 2).

${ }^{40}$ The third possibility, namely, that only quality of the match is observed (or can be used in court) does not help here: since all consumers buy for all signal realizations, the ex post distribution of quality is the same as the prior one. This would be different if consumers buy only above a certain threshold as in Section 4.
} 


$$
\widehat{\beta}=\frac{1}{N} \sum_{i=1}^{N} s_{i}-\mu
$$

The authority tests $H_{0}: \beta=0$ against $H_{1}: \beta>0$ and punishes the seller only when $H_{0}$ is rejected. Since $s_{i} \rightsquigarrow \mathcal{N}\left(\mu+\beta, \sigma^{2}+\eta^{2}\right)$, the estimator $\widehat{\beta}$ is distributed as $\widehat{\beta} \rightsquigarrow \mathcal{N}\left(\beta, \frac{\sigma^{2}+\eta^{2}}{N}\right)$. It has a higher variance, $\frac{\sigma^{2}+\eta^{2}}{N}$, than the one in Section $2.3, \frac{\eta^{2}}{N}$, since the authority does not observe the match quality. The costs are

$$
C(\beta, \eta)=\beta\left(1-\Phi\left(z_{\alpha}-\frac{\beta \sqrt{N}}{\sqrt{\sigma^{2}+\eta^{2}}}\right)\right)+\frac{\sqrt{\sigma^{2}+\eta^{2}}}{\sqrt{N}} \phi\left(z_{\alpha}-\frac{\beta \sqrt{N}}{\sqrt{\sigma^{2}+\eta^{2}}}\right)
$$

It turns out that the seller's problem is not concave with this cost function unless there are high enough punitive damages $d$ as in Section 5.4.

Proposition 7 If only signals are observed (and there are punitive damages $d$ ), the equilibrium bias and noise are

$$
\begin{aligned}
\beta_{s}^{*} & =\frac{\sigma}{\sqrt{N}} \frac{1}{z_{\alpha}} \sqrt{\max \left\{\kappa_{d}, 0\right\}+1} \\
\eta_{s}^{*} & =\sigma \sqrt{\max \left\{\kappa_{d}, 0\right\}}
\end{aligned}
$$

The equilibrium bias is higher, $\beta_{s}^{*}>\beta_{d}^{*}$, and the noise is the same, $\eta_{s}^{*}=\eta_{d}^{*}$, as in the case when both signals and match qualities are observed.

Proof. Similar to the proof of Proposition 1.

The equilibrium bias increases since it is now more difficult to convict the seller. The equilibrium noise does not change. This perhaps counterintuitive result has the same explanation as the one of the equilibrium noise not depending on the sample size $N$ in Corollary 1. The minimum costs with respect to the noise are obtained when $\frac{\beta \sqrt{N}}{\sqrt{\sigma^{2}+\eta^{2}}}=\frac{1}{z_{\alpha}}$. The noise is then found from $\frac{\sigma^{2}}{\sigma^{2}+\eta^{2}}=d\left(1-\Phi\left(z_{\alpha}-\frac{1}{z_{\alpha}}\right)+z_{\alpha} \phi\left(z_{\alpha}-\frac{1}{z_{\alpha}}\right)\right)$, where the right hand side is the equilibrium marginal costs of bias that do not depend on $\sigma^{2}$.

\subsection{Natural noise in the communication}

Suppose there is a natural noise in the communication $v^{2}$. For example, the communication is always imperfect unless the seller makes it better, possibly, at some effort costs. Then, the error term $\varepsilon$ is distributed as $\varepsilon \rightsquigarrow \mathcal{N}\left(\beta, v^{2}+\eta^{2}\right)$. Let us consider 
two polar cases. First, suppose that the effort costs are zero, that is, the seller can set $\eta^{2}$ equal to $-v^{2}$ making communication perfect at no cost. The seller's problem with respect to noise can be thought of as choosing the total variance $v^{2}+\eta^{2}$ and its equilibrium level will be the same as in baseline model (5b), that is, $\sqrt{v^{2}+\eta^{* 2}}=\sigma \sqrt{\kappa}$. The equilibrium bias will also be the same as in the baseline model (5a).

The opposite case is when the effort costs are so high that the seller never chooses a negative $\eta^{2}$. The seller's problem with respect to noise is choosing the total variance $v^{2}+\eta^{2}$ with the constraint $\eta^{2} \geq 0$. If the (unconstrained) equilibrium noise $\eta^{*}(5 \mathrm{~b})$ is higher than $v^{2}$, then this constraint is not binding and the equilibrium is not affected. Otherwise, the equilibrium becomes $\eta^{*}=0$ (that is, the variance of the error term is at its lowest level $v^{2}$ ) and the equilibrium bias is found from the first-order condition (12a) when $\eta=v$.

\subsection{One-stage procedure used by the authority}

The procedure used by the authority in the baseline model of Section 2 is probably best interpreted as a two-stage procedure. At the first stage, the authority determines if the seller is guilty, and if it is the case, the authority fixes the punishment at the second stage. For this reason, the fine depends only on the estimated bias but not on the noise.

If the authority were to determine both if the seller is guilty and the size of the fine at the same stage, it then might account for the noise when fixing the fine, that is, to fine the seller by $\frac{\widehat{\beta}}{\eta / \sqrt{N}}$. The costs are then

$C(\beta, \eta)=\int_{z_{\alpha} \frac{\eta}{\sqrt{N}}}^{+\infty} \widehat{\beta} \frac{\sqrt{N}}{\eta} f(\widehat{\beta}) d \widehat{\beta}=\beta \frac{\sqrt{N}}{\eta}\left(1-\Phi\left(z_{\alpha}-\frac{\beta \sqrt{N}}{\eta}\right)\right)+\phi\left(z_{\alpha}-\frac{\beta \sqrt{N}}{\eta}\right)$.

The costs now depend only on the ratio $\frac{\beta}{\eta}$. A higher noise decreases both the probability of conviction and the fine whenever it is imposed. Therefore, the seller will always introduce maximum possible noise. This observation leads to the next proposition.

Proposition 8 If the authority adjusts the fine for the noise, there is no information transmission in the equilibrium.

Proof. The first derivative of the profit function with respect to the noise is

$$
\frac{\partial \Pi}{\partial \eta}=-(\beta-\widetilde{\beta}) \frac{2 \eta \sigma^{2}}{\left(\sigma^{2}+\eta^{2}\right)^{2}}+\frac{\beta \sqrt{N}}{\eta^{2}}\left(1-\Phi\left(z_{\alpha}-\frac{\beta \sqrt{N}}{\eta}\right)+z_{\alpha} \phi\left(z_{\alpha}-\frac{\beta \sqrt{N}}{\eta}\right)\right)
$$


In the equilibrium the buyers are not misled, $\beta=\widetilde{\beta}$, and this derivative is positive. The seller introduces then infinite noise, $\eta=+\infty$, and the buyers ignore the seller's signal.

\section{Conclusion}

In this paper we investigated seller's incentives to provide (un)biased and (un)informative advice and the resulting equilibrium communication. We found that the biasing the advice and making it more noisy are complements: the seller employs says either an exact truth or a vague lie. For example, a higher buyers' heterogeneity, a higher standard of proof employed by the authority and a higher share of credulous consumers make the signal sent by the seller to the buyers more biased and less precise.

An interesting direction for future work is to characterize the optimal policy of the authority. In the model, a policy is described by three parameters: the number of consumers sampled $N$, the standard of proof used when establishing if the seller is guilty $z_{\alpha}$ and the size of the fine in relation to the estimated bias $d$. We have taken these parameters as given and found that the comparative statics are intuitive: a higher $N$, a lower $z_{\alpha}$ and a higher $d$ reduce the equilibrium bias. This poses the question of why the authority does not set them up at the level that would completely deter the seller from biasing the signal. The reason is that there are of course costs of a strict policy. Surveying a lot of consumers, that is, a high $N$, is costly in monetary terms; a lower standard of proof $z_{\alpha}$ makes conviction of innocent sellers more likely and higher punitive damages $d$ make innocent sellers pay a higher fine. Extending the model to incorporate these costs may produce relevant and realistic recommendations on the optimal policy.

\section{Appendix}

Proof of Proposition 1. The first-order conditions of the seller's problem with respect to $\beta$ and $\eta$, respectively, are

$$
\begin{array}{r}
\frac{\sigma^{2}}{\sigma^{2}+\eta^{2}}-\left(1-\Phi\left(z_{\alpha}-\frac{\beta \sqrt{N}}{\eta}\right)+z_{\alpha} \phi\left(z_{\alpha}-\frac{\beta \sqrt{N}}{\eta}\right)\right)=0 \\
-(\beta-\widetilde{\beta}) \frac{2 \eta \sigma^{2}}{\left(\sigma^{2}+\eta^{2}\right)^{2}}-\frac{1}{\sqrt{N}} \phi\left(z_{\alpha}-\frac{\beta \sqrt{N}}{\eta}\right)\left[1-\frac{\beta \sqrt{N}}{\eta} z_{\alpha}\right]=0
\end{array}
$$


In the equilibrium $\beta=\widetilde{\beta}$ and from (12b) $\frac{\beta \sqrt{N}}{\eta}=\frac{1}{z_{\alpha}}$. Plug this into (12a) to obtain $\beta^{*}$ and then $\eta^{*}$.

To check the second-order conditions, differentiate (12a) with respect to $\beta$ to obtain $\frac{\partial^{2} \Pi}{\partial \beta^{2}}$ and $(12 \mathrm{~b})$ with respect to $\eta$ and $\beta$ to obtain $\frac{\partial^{2} \Pi}{\partial \eta^{2}}$ and $\frac{\partial^{2} \Pi}{\partial \eta \partial \beta}$, respectively:

$$
\begin{aligned}
\frac{\partial^{2} \Pi}{\partial \beta^{2}} & =-\frac{\sqrt{N}}{\eta} \phi\left(z_{\alpha}-\frac{\beta \sqrt{N}}{\eta}\right)\left(1-\frac{\beta \sqrt{N}}{\eta} z_{\alpha}+z_{\alpha}^{2}\right) \\
\frac{\partial^{2} \Pi}{\partial \eta^{2}} & =-(\beta-\widetilde{\beta}) \frac{\sigma^{2}-3 \eta^{2}}{\left(\sigma^{2}+\eta^{2}\right)^{3}}-\frac{\beta^{2} \sqrt{N}}{\eta^{3}} \phi\left(z_{\alpha}-\frac{\beta \sqrt{N}}{\eta}\right)\left(1-\frac{\beta \sqrt{N}}{\eta} z_{\alpha}+z_{\alpha}^{2}\right) \\
\frac{\partial^{2} \Pi}{\partial \eta \partial \beta} & =-\frac{2 \eta \sigma^{2}}{\left(\sigma^{2}+\eta^{2}\right)^{2}}+\frac{\beta \sqrt{N}}{\eta^{2}} \phi\left(z_{\alpha}-\frac{\beta \sqrt{N}}{\eta}\right)\left(1-\frac{\beta \sqrt{N}}{\eta} z_{\alpha}+z_{\alpha}^{2}\right)
\end{aligned}
$$

In the equilibrium $\beta=\beta^{*}=\widetilde{\beta}$ and $\frac{\beta^{*} \sqrt{N}}{\eta^{*}}=\frac{1}{z_{\alpha}}$ so these derivatives become

$$
\begin{aligned}
\frac{\partial^{2} \Pi}{\partial \beta^{2}} & =-\frac{\sqrt{N}}{\eta^{*}} \phi\left(z_{\alpha}-\frac{1}{z_{\alpha}}\right) z_{\alpha}^{2}<0 \\
\frac{\partial^{2} \Pi}{\partial \eta^{2}} & =-\frac{\beta^{* 2} \sqrt{N}}{\eta^{* 3}} \phi\left(z_{\alpha}-\frac{1}{z_{\alpha}}\right) z_{\alpha}^{2}<0 \\
\frac{\partial^{2} \Pi}{\partial \eta \partial \beta} & =-\frac{2 \eta^{*} \sigma^{2}}{\left(\sigma^{2}+\eta^{* 2}\right)^{2}}+\frac{\beta^{*} \sqrt{N}}{\eta^{* 2}} \phi\left(z_{\alpha}-\frac{1}{z_{\alpha}}\right) z_{\alpha}^{2}
\end{aligned}
$$

Check that the determinant of the Hessian is positive:

$$
\begin{aligned}
\frac{\partial^{2} \Pi}{\partial \beta^{2}} \frac{\partial^{2} \Pi}{\partial \eta^{2}}-\left(\frac{\partial^{2} \Pi}{\partial \eta \partial \beta}\right)^{2}= & \frac{\beta^{* 2} N}{\eta^{* 4}} \phi^{2}\left(z_{\alpha}-\frac{1}{z_{\alpha}}\right) z_{\alpha}^{4}-\left(\frac{2 \eta^{*} \sigma^{2}}{\left(\sigma^{2}+\eta^{* 2}\right)^{2}}\right)^{2} \\
& +\frac{4 \eta^{*} \sigma^{2}}{\left(\sigma^{2}+\eta^{* 2}\right)^{2}} \frac{\beta^{*} \sqrt{N}}{\eta^{2}} \phi\left(z_{\alpha}-\frac{1}{z_{\alpha}}\right) z_{\alpha}^{2}-\frac{\beta^{* 2} N}{\eta^{* 4}} \phi^{2}\left(z_{\alpha}-\frac{1}{z_{\alpha}}\right) z_{\alpha}^{4} \\
= & \frac{4 \sigma^{2}}{\left(\sigma^{2}+\eta^{* 2}\right)^{2}}\left(\frac{\beta^{*} \sqrt{N}}{\eta^{* 2}} \phi\left(z_{\alpha}-\frac{1}{z_{\alpha}}\right) z_{\alpha}^{2}-\frac{\eta^{*} \sigma^{2}}{\left(\sigma^{2}+\eta^{* 2}\right)^{2}}\right) \\
= & \frac{4 \sigma^{2}}{\eta^{*}\left(\sigma^{2}+\eta^{* 2}\right)^{2}}\left(\phi\left(z_{\alpha}-\frac{1}{z_{\alpha}}\right) z_{\alpha}-\frac{\kappa}{(1+\kappa)^{2}}\right)
\end{aligned}
$$

It is positive for $z_{\alpha}<2.436$, i.e., $\alpha>0.75 \%$.

These are only local second-order conditions. In our candidate equilibrium, i.e., if the seller's bias and buyer's conjecture are (5a) and the noise is (5b), the seller earns $\mu$ minus the costs. Then, he might prefer to deviate in the following way: stop the communication by introducing infinite noise, in which case the buyer disregards the signal and the revenues are still $\mu$, and introduce an infinite negative bias in 
order to drive the costs to zero. ${ }^{41}$ Usually, however, the seller is obliged to provide some minimal amount of information about the product; in other words, there is an upper bound on $\eta$. While a small negative bias can be interpreted as "modesty", an infinite one is clearly unrealistic. We thus assume that an upper bound on noise and a lower bound on bias are such that this deviation is not profitable and (5a-5b) is an equilibrium.

Proof of Proposition 2. The first-order conditions of the seller's problem with respect to $\beta$ and $\eta$, respectively, are

$$
\begin{aligned}
& \mu g(\mu+\widetilde{\beta})-\left(1-\Phi\left(z_{\alpha}-\frac{\beta \sqrt{N}}{\eta}\right)+z_{\alpha} \phi\left(z_{\alpha}-\frac{\beta \sqrt{N}}{\eta}\right)\right)=0 \\
& -\mu \eta g(\mu+\widetilde{\beta}) \frac{\beta-\widetilde{\beta}}{\sigma^{2}+\eta^{2}}-\frac{1}{\sqrt{N}} \phi\left(z_{\alpha}-\frac{\beta \sqrt{N}}{\eta}\right)\left[1-\frac{\beta \sqrt{N}}{\eta} z_{\alpha}\right]=0
\end{aligned}
$$

In the equilibrium, $\beta=\widetilde{\beta}$. From (13b) $\frac{\beta \sqrt{N}}{\eta}=\frac{1}{z_{\alpha}}$ as in Section 3. Plug this into (13a) to obtain $\beta_{p}^{*}$ and then $\eta_{p}^{*}$.

To check the second-order conditions, we differentiate (13a) with respect to $\beta$ and $\eta$ to obtain $\frac{\partial^{2} \Pi}{\partial \beta^{2}}$ and $\frac{\partial^{2} \Pi}{\partial \eta \partial \beta}$, respectively and (13b) with respect to $\eta$ to obtain $\frac{\partial^{2} \Pi}{\partial \eta^{2}}$ :

$$
\begin{aligned}
\frac{\partial^{2} \Pi}{\partial \beta^{2}}= & \mu g(\mu+\widetilde{\beta}) \frac{\widetilde{\beta}-\beta}{\sigma^{2}+\eta^{2}}-\frac{\sqrt{N}}{\eta} \phi\left(z_{\alpha}-\frac{\beta \sqrt{N}}{\eta}\right)\left(1+z_{\alpha}\left(z_{\alpha}-\frac{\beta \sqrt{N}}{\eta}\right)\right) \\
\frac{\partial^{2} \Pi}{\partial \eta^{2}}= & -\mu g(\mu+\widetilde{\beta}) \frac{\beta-\widetilde{\beta}}{\sigma^{2}+\eta^{2}}\left[1+\eta^{2}\left(\frac{(\widetilde{\beta}-\beta)^{2}-3\left(\sigma^{2}+\eta^{2}\right)}{\left(\sigma^{2}+\eta^{2}\right)^{2}}\right)\right] \\
& -\frac{\beta^{2}}{\eta^{2}} \frac{\sqrt{N}}{\eta} \phi\left(z_{\alpha}-\frac{\beta \sqrt{N}}{\eta}\right)\left[1+z_{\alpha}\left(z_{\alpha}-\frac{\beta \sqrt{N}}{\eta}\right)\right] \\
\frac{\partial^{2} \Pi}{\partial \eta \partial \beta}= & \mu \eta g(\mu+\widetilde{\beta}) \frac{(\widetilde{\beta}-\beta)^{2}-\left(\sigma^{2}+\eta^{2}\right)}{\left(\sigma^{2}+\eta^{2}\right)^{2}}+\frac{\beta \sqrt{N}}{\eta^{2}} \phi\left(z_{\alpha}-\frac{\beta \sqrt{N}}{\eta}\right)\left(1+z_{\alpha}\left(z_{\alpha}-\frac{\beta \sqrt{N}}{\eta}\right)\right)
\end{aligned}
$$

\footnotetext{
${ }^{41}$ For a negative or zero bias the costs are strictly increasing in the noise, see the proof of Lemma 1.
} 
In equilibrium, $\beta=\beta_{p}^{*}=\widetilde{\beta}$ and $\frac{\beta_{p}^{*} \sqrt{N}}{\eta_{p}^{*}}=\frac{1}{z_{\alpha}}$ so these derivatives become:

$$
\begin{aligned}
\frac{\partial^{2} \Pi}{\partial \beta^{2}} & =-\frac{z_{\alpha}^{2} \sqrt{N}}{\eta_{p}^{*}} \phi\left(z_{\alpha}-\frac{1}{z_{\alpha}}\right)<0 \\
\frac{\partial^{2} \Pi}{\partial \eta^{2}} & =-\frac{z_{\alpha} \beta_{p}^{*}}{\eta_{p}^{* 2}} \phi\left(z_{\alpha}-\frac{1}{z_{\alpha}}\right)<0 \\
\frac{\partial^{2} \Pi}{\partial \eta \partial \beta} & =-\mu \eta_{p}^{*} g\left(\mu+\beta_{p}^{*}\right) \frac{1}{\sigma^{2}+\eta_{p}^{* 2}}+\frac{z_{\alpha}}{\eta_{p}^{*}} \phi\left(z_{\alpha}-\frac{1}{z_{\alpha}}\right)
\end{aligned}
$$

The determinant of the Hessian, $\frac{\partial^{2} \Pi}{\partial \beta^{2}} \frac{\partial^{2} \Pi}{\partial \eta^{2}}-\left(\frac{\partial^{2} \Pi}{\partial \eta \partial \beta}\right)^{2}$, is equal to

$$
\begin{aligned}
& \frac{z_{\alpha}^{2} \sqrt{N}}{\eta_{p}^{*}} \frac{z_{\alpha} \beta_{p}^{*}}{\eta_{p}^{* 2}} \phi\left(z_{\alpha}-\frac{1}{z_{\alpha}}\right)^{2}-\left(\frac{z_{\alpha}}{\eta_{p}^{*}} \phi\left(z_{\alpha}-\frac{1}{z_{\alpha}}\right)-\mu \eta_{p}^{*} g\left(\mu+\beta_{p}^{*}\right) \frac{1}{\sigma^{2}+\eta_{p}^{* 2}}\right)^{2} \\
= & 2 \frac{\mu g\left(\mu+\beta_{p}^{*}\right)}{\sigma^{2}+\eta_{p}^{* 2}} z_{\alpha} \phi\left(z_{\alpha}-\frac{1}{z_{\alpha}}\right)-\left(\frac{\mu \eta_{p}^{*} g\left(\mu+\beta_{p}^{*}\right)}{\sigma^{2}+\eta_{p}^{* 2}}\right)^{2} \\
= & \frac{\mu}{\sqrt{2 \pi\left(\sigma^{2}+\eta_{p}^{* 2}\right)}\left(\sigma^{2}+\eta_{p}^{* 2}\right)}\left[2 z_{\alpha} \phi\left(z_{\alpha}-\frac{1}{z_{\alpha}}\right)-\frac{\eta_{p}^{* 2} \lambda}{\sigma^{2}+\eta_{p}^{* 2}}\right] \\
= & \frac{2 \pi \sigma^{2} \lambda^{3}-\mu^{2}\left[1-\Phi\left(z_{\alpha}-\frac{1}{z_{\alpha}}\right)-z_{\alpha} \phi\left(z_{\alpha}-\frac{1}{z_{\alpha}}\right)\right]}{\mu \sqrt{2 \pi}\left(\sigma^{2}+\eta_{p}^{* 2}\right)^{\frac{3}{2}}}
\end{aligned}
$$

Its sign is the one of the numerator. In Figure 2 we plot the parameter range for which it is positive and, therefore, the second-order conditions are satisfied. In particular, they are satisfied if $z_{\alpha} \geq 1.09$ (i.e., $\alpha \leq 13.79 \%$ ), in which case $1-\Phi\left(z_{\alpha}-\frac{1}{z_{\alpha}}\right)-$ $z_{\alpha} \phi\left(z_{\alpha}-\frac{1}{z_{\alpha}}\right)<0$.

Proof of Corollary 2. Consider the effect of $z_{\alpha}$. Since $\frac{\partial \lambda}{\partial z_{\alpha}}=-z_{\alpha}^{2} \phi\left(z_{\alpha}-\frac{1}{z_{\alpha}}\right)<0$, $\frac{\partial \eta_{p}^{*}}{\partial z_{\alpha}}>0$. The effect on the equilibrium bias is more involved:

$$
\frac{\partial \beta_{p}^{*}}{\partial z_{\alpha}}=\frac{\sqrt{\frac{\mu^{2}}{2 \pi \lambda^{2}}-\sigma^{2}}}{z_{\alpha}^{2} \sqrt{N}}\left[\frac{\mu^{2} z_{\alpha}^{3} \phi\left(z_{\alpha}-\frac{1}{z_{\alpha}}\right)}{\lambda\left(\mu^{2}-2 \pi \lambda^{2} \sigma^{2}\right)}-1\right] .
$$

Since $\frac{\mu^{2}}{\sigma^{2} 2 \pi}>\lambda^{2}$ for the bias to be positive, $\frac{\partial \beta}{\partial z_{\alpha}}$ is positive if:

$$
2 \pi \lambda^{3} \sigma^{2}>\mu^{2}\left[\lambda-z_{\alpha}^{3} \phi\left(z_{\alpha}-\frac{1}{z_{\alpha}}\right)\right] .
$$




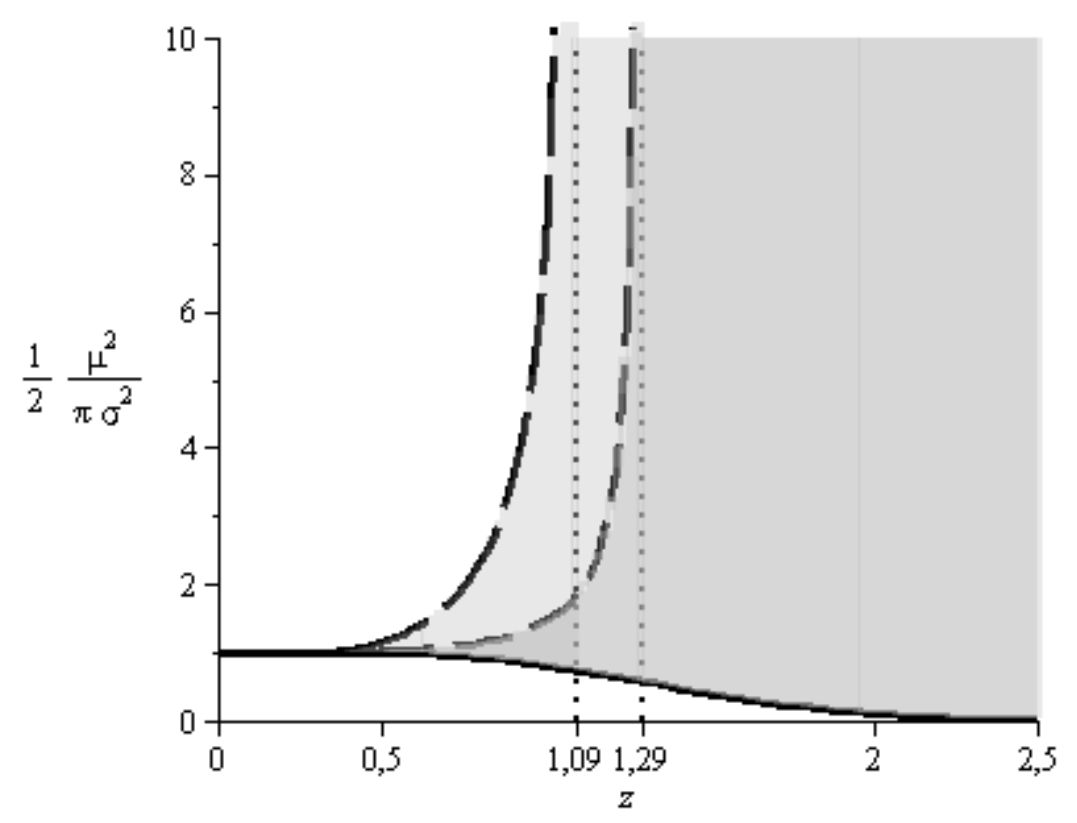

Figure 2: In both shaded areas the second-order conditions are satisfied and $\beta_{p}^{*}$ and $\eta_{p}^{*}$ are positive, that is, $\frac{\mu^{2}}{2 \pi \lambda^{2}}-\sigma^{2}>0$. In the dark shaded area $\frac{\partial \beta_{p}^{*}}{\partial z_{\alpha}}$ is positive.

In Figure 2 we plot the parameter range for which this inequality holds. In particular, it holds if $z_{\alpha} \geq 1.29$ (i.e., $\alpha \leq 9.85 \%$ ) in which case $\lambda-z_{\alpha}^{3} \phi\left(z_{\alpha}-\frac{1}{z_{\alpha}}\right)<0$.

The remaining comparative statics are straightforward.

Proof of Proposition 3. The first-order conditions of the seller's problem with respect to $\beta$ and $\eta$, respectively, are

$$
\begin{aligned}
p g(\bar{s})-\left(1-\Phi\left(z_{\alpha}-\frac{\beta \sqrt{N}}{\eta}\right)+z_{\alpha} \phi\left(z_{\alpha}-\frac{\beta \sqrt{N}}{\eta}\right)\right) & =0(15 \mathrm{a}) \\
-p \eta g(\bar{s})\left[\frac{\beta-\widetilde{\beta}}{\sigma^{2}+\eta^{2}}+\frac{p-\mu}{\sigma^{2}}\right]-\frac{1}{\sqrt{N}} \phi\left(z_{\alpha}-\frac{\beta \sqrt{N}}{\eta}\right)\left[1-\frac{\beta \sqrt{N}}{\eta} z_{\alpha}\right] & =0(15 \mathrm{~b})
\end{aligned}
$$

where $\bar{s}=p+\widetilde{\beta}+(p-\mu) \frac{\eta^{2}}{\sigma^{2}}$. In the equilibrium, $\beta=\widetilde{\beta}$. If $p>(<) \mu$ then $\frac{1}{\sqrt{N} z_{\alpha}}<(>) \frac{\beta}{\eta}$ in order for $(15 \mathrm{~b})$ to hold.

Derivation of the buyer's posterior in Section 5.2. See Theil (1971), ch. 4.7, for the details of the derivation of posterior in the multivariate normal case. 
Let us write the covariance matrix of $\theta_{1}, s_{1}$ and $s_{2}$. Using

$\operatorname{Cov}\left(\theta_{1}, s_{1}\right)=\operatorname{Cov}\left(\theta_{1}, \theta_{1}+\varepsilon_{1}\right)=\operatorname{Var}\left(\theta_{1}\right)=\sigma_{1}^{2}$

$\operatorname{Cov}\left(\theta_{1}, s_{2}\right)=\operatorname{Cov}\left(\theta_{1}, \theta_{2}+\varepsilon_{2}\right)=\operatorname{Cov}\left(\theta_{1}, \theta_{2}\right)=\rho_{\theta} \sigma_{1} \sigma_{2}$

$\operatorname{Cov}\left(s_{1}, s_{2}\right)=\operatorname{Cov}\left(\theta_{1}+\varepsilon_{1}, \theta_{2}+\varepsilon_{2}\right)=\operatorname{Cov}\left(\theta_{1}, \theta_{2}\right)+\operatorname{Cov}\left(\varepsilon_{1}, \varepsilon_{2}\right)=\rho_{\theta} \sigma_{1} \sigma_{2}+\rho_{\varepsilon} \eta_{1} \eta_{2}$

the matrix is

\begin{tabular}{c|ccc} 
& $\theta_{1}$ & $s_{1}$ & $s_{2}$ \\
\hline$\theta_{1}$ & $\sigma_{1}^{2}$ & $\sigma_{1}^{2}$ & $\rho_{\theta} \sigma_{1} \sigma_{2}$ \\
$s_{1}$ & $\sigma_{1}^{2}$ & $\sigma_{1}^{2}+\eta_{1}^{2}$ & $\rho_{\theta} \sigma_{1} \sigma_{2}+\rho_{\varepsilon} \eta_{1} \eta_{2}$ \\
$s_{2}$ & $\rho_{\theta} \sigma_{1} \sigma_{2}$ & $\rho_{\theta} \sigma_{1} \sigma_{2}+\rho_{\varepsilon} \eta_{1} \eta_{2}$ & $\sigma_{2}^{2}+\eta_{2}^{2}$
\end{tabular}

Denote the covariance matrix of $s_{1}$ and $s_{2}$ as $\Sigma$ and find its inverse:

$$
\Sigma^{-1}=\frac{1}{\operatorname{det}(\Sigma)}\left(\begin{array}{cc}
\sigma_{2}^{2}+\eta_{2}^{2} & -\rho_{\theta} \sigma_{1} \sigma_{2}-\rho_{\varepsilon} \eta_{1} \eta_{2} \\
-\rho_{\theta} \sigma_{1} \sigma_{2}-\rho_{\varepsilon} \eta_{1} \eta_{2} & \sigma_{1}^{2}+\eta_{1}^{2}
\end{array}\right)
$$

where $\operatorname{det}(\Sigma)=\left(\sigma_{2}^{2}+\eta_{2}^{2}\right)\left(\sigma_{1}^{2}+\eta_{1}^{2}\right)-\left(\rho_{\theta} \sigma_{1} \sigma_{2}+\rho_{\varepsilon} \eta_{1} \eta_{2}\right)^{2}$. The expectation of $\theta_{1}$ conditional on $s_{1}$ and $s_{2}, E\left[\theta_{1} \mid s_{1}, s_{2}, \eta_{1}, \eta_{2}, \widetilde{\beta}_{1}, \widetilde{\beta}_{2}\right]$, equals to

$$
\begin{aligned}
& \mu_{1}+\left(\begin{array}{ll}
\sigma_{1}^{2} & \rho_{\theta} \sigma_{1} \sigma_{2}
\end{array}\right) \Sigma^{-1}\left(\begin{array}{c}
s_{1}-\mu_{1}-\widetilde{\beta}_{1} \\
s_{2}-\mu_{2}-\widetilde{\beta}_{2}
\end{array}\right) \\
= & \mu_{1}+\frac{1}{\operatorname{det}(\Sigma)}\left(\begin{array}{c}
\sigma_{1}^{2}\left(\sigma_{2}^{2}+\eta_{2}^{2}\right)-\rho_{\theta} \sigma_{1} \sigma_{2}\left(\rho_{\theta} \sigma_{1} \sigma_{2}+\rho_{\varepsilon} \eta_{1} \eta_{2}\right) \\
-\sigma_{1}^{2}\left(\rho_{\theta} \sigma_{1} \sigma_{2}+\rho_{\varepsilon} \eta_{1} \eta_{2}\right)+\rho_{\theta} \sigma_{1} \sigma_{2}\left(\sigma_{1}^{2}+\eta_{1}^{2}\right)
\end{array}\right)\left(\begin{array}{c}
s_{1}-\mu_{1}-\widetilde{\beta}_{1} \\
s_{2}-\mu_{2}-\widetilde{\beta}_{2}
\end{array}\right) \\
= & \mu_{1}+\frac{\sigma_{1}^{2}\left(\sigma_{2}^{2}+\eta_{2}^{2}\right)-\rho_{\theta} \sigma_{1} \sigma_{2}\left(\rho_{\theta} \sigma_{1} \sigma_{2}+\rho_{\varepsilon} \eta_{1} \eta_{2}\right)}{\left(\sigma_{2}^{2}+\eta_{2}^{2}\right)\left(\sigma_{1}^{2}+\eta_{1}^{2}\right)-\left(\rho_{\theta} \sigma_{1} \sigma_{2}+\rho_{\varepsilon} \eta_{1} \eta_{2}\right)^{2}}\left(s_{1}-\mu_{1}-\widetilde{\beta}_{1}\right) \\
& +\frac{-\sigma_{1}^{2} \rho_{\varepsilon} \eta_{1} \eta_{2}+\rho_{\theta} \sigma_{1} \sigma_{2} \eta_{1}^{2}}{\left(\sigma_{2}^{2}+\eta_{2}^{2}\right)\left(\sigma_{1}^{2}+\eta_{1}^{2}\right)-\left(\rho_{\theta} \sigma_{1} \sigma_{2}+\rho_{\varepsilon} \eta_{1} \eta_{2}\right)^{2}}\left(s_{2}-\mu_{2}-\widetilde{\beta}_{2}\right) \\
= & \mu_{1}+\frac{1+\frac{\eta_{2}^{2}}{\sigma_{2}^{2}}-\rho_{\theta}\left(\rho_{\theta}+\rho_{\varepsilon} \frac{\eta_{1} \eta_{2}}{\sigma_{1} \sigma_{2}}\right)}{\left(1+\frac{\eta_{2}^{2}}{\sigma_{2}^{2}}\right)\left(1+\frac{\eta_{1}^{2}}{\sigma_{1}^{2}}\right)-\left(\rho_{\theta}+\rho_{\varepsilon} \frac{\eta_{1} \eta_{2}}{\sigma_{1} \sigma_{2}}\right)^{2}}\left(s_{1}-\mu_{1}-\widetilde{\beta}_{1}\right) \\
& +\frac{\rho_{\theta} \frac{\eta_{1}^{2}}{\sigma_{1} \sigma_{2}}-\rho_{\varepsilon} \frac{\eta_{1} \eta_{2}}{\sigma_{2}^{2}}}{\left(1+\frac{\eta_{2}^{2}}{\sigma_{2}^{2}}\right)\left(1+\frac{\eta_{1}^{2}}{\sigma_{1}^{2}}\right)-\left(\rho_{\theta}+\rho_{\varepsilon} \frac{\eta_{1} \eta_{2}}{\sigma_{1} \sigma_{2}}\right)^{2}}\left(s_{2}-\mu_{2}-\widetilde{\beta}_{2}\right) .
\end{aligned}
$$

\section{Proof of Proposition 4.}

Seller 1 maximizes revenues (10) minus costs (4b) taking $\eta_{2}$ as given. In the 
equilibrium $\beta_{1}=\widetilde{\beta}_{1}$ and, as in the proof of Proposition $1, \frac{\beta_{1} \sqrt{N}}{\eta_{1}}=\frac{1}{z_{\alpha}}$. The first-order condition with respect to the bias becomes

$$
\frac{1+\frac{\eta_{2}^{2}}{\sigma_{2}^{2}}-\rho_{\theta}\left(\rho_{\theta}+\rho_{\varepsilon} \frac{\eta_{1} \eta_{2}}{\sigma_{1} \sigma_{2}}\right)}{\left(1+\frac{\eta_{2}^{2}}{\sigma_{2}^{2}}\right)\left(1+\frac{\eta_{1}^{2}}{\sigma_{1}^{2}}\right)-\left(\rho_{\theta}+\rho_{\varepsilon} \frac{\eta_{1} \eta_{2}}{\sigma_{1} \sigma_{2}}\right)^{2}}=1-\Phi\left(z_{\alpha}-\frac{1}{z_{\alpha}}\right)+z_{\alpha} \phi\left(z_{\alpha}-\frac{1}{z_{\alpha}}\right) .
$$

Note that (16) is symmetric in "normalized" noises $\frac{\eta_{1}}{\sigma_{1}}$ and $\frac{\eta_{2}}{\sigma_{2}}$. To find symmetric equilibria, denote $\widetilde{\eta}=\frac{\eta_{1}}{\sigma_{1}}=\frac{\eta_{2}}{\sigma_{2}}$ and rewrite (16) as

$$
\left(1-\rho_{\varepsilon}^{2}\right) \widetilde{\eta}^{4}-(\kappa-1)\left(1-\rho_{\theta} \rho_{\varepsilon}\right) \widetilde{\eta}^{2}-\kappa\left(1-\rho_{\theta}^{2}\right)=0
$$

which is a quadratic equation in $\widetilde{\eta}^{2}$ (unless $\left.\rho_{\varepsilon}= \pm 1\right)(\kappa$ is defined in Proposition 1 ). It has two roots of opposite signs and the positive root is

$$
\widetilde{\eta}^{2}=\frac{(\kappa-1)\left(1-\rho_{\theta} \rho_{\varepsilon}\right)+\sqrt{(\kappa-1)^{2}\left(1-\rho_{\theta} \rho_{\varepsilon}\right)^{2}+4 \kappa\left(1-\rho_{\theta}^{2}\right)\left(1-\rho_{\varepsilon}^{2}\right)}}{2\left(1-\rho_{\varepsilon}^{2}\right)} .
$$

Considering the cases of $\rho_{\varepsilon}= \pm 1$ and $\rho_{\theta}= \pm 1$ is straightforward.

We could not prove the absence of asymmetric equilibria; however, we have not encountered them in many numerical examples that we plotted. ${ }^{42}$

The second-order conditions are difficult to check but they are satisfied, by continuity, at least for $\rho_{\varepsilon}$ and $\rho_{\theta}$ close to zero.

\section{References}

Anderson, A., And L. M. B. Cabral (2007): "Go for broke or play it safe? Dynamic competition with choice of variance," The RAND Journal of Economics, 38(3), 593-609.

Armstrong, M. (2008): "Interactions between Competition and Consumer Policy," Competition Policy International, 4(1), 96-147.

Blume, A., And O. Board (2010): "Intentional Vagueness," http://www.pitt.edu/ ojboard/papers/vagueness.pdf.

\footnotetext{
${ }^{42}$ To find asymmetric equilibria (or to prove that they do not exist), denote $\widetilde{\eta}_{1}=\frac{\eta_{1}}{\sigma_{1}}$ and $\widetilde{\eta}_{2}=\frac{\eta_{2}}{\sigma_{2}}$. The reaction curve $\widetilde{\eta}_{1}\left(\widetilde{\eta}_{2}\right)$ can be found from rewriting (16) as

$$
\widetilde{\eta}_{1}^{2}\left(1+\widetilde{\eta}_{2}^{2}\left(1-\rho_{\varepsilon}^{2}\right)\right)+(\kappa-1) \widetilde{\eta}_{1} \widetilde{\eta}_{2} \rho_{\theta} \rho_{\varepsilon}-\kappa\left(1-\rho_{\theta}^{2}+\widetilde{\eta}_{2}^{2}\right)=0 .
$$
}

Equilibria are its intersections with the (symmetric) reaction curve $\widetilde{\eta}_{2}\left(\widetilde{\eta}_{1}\right)$. 
Casella, G., and R. L. Berger (2002): Statistical inference. Duxbury Press, Belmont.

Chakraborty, A., and R. Harbaugh (2010): "Persuasion by Cheap Talk," American Economic Review, 100(5), 2361-82.

Crawford, V. P., and J. Sobel (1982): "Strategic Information Transmission," Econometrica, 50(6), 1431-1451.

Davis, M. L. (1994): "The Value of Truth and the Optimal Standard of Proof in Legal Disputes," Journal of Law, Economics, and Organization, 10(2), 343-359.

Dewatripont, M., I. Jewitt, and J. Tirole (1999): "The Economics of Career Concerns, Part I: Comparing Information Structures," The Review of Economic Studies, 66(1), 183-198.

FTC (2008): "Before the Board of Governors of the Federal Reserve System: In the Matter of Request for Comments on Truth in Lending, Proposed Rule Docket No. R-1305," Available at http://www.federalreserve.gov/SECRS/2008/April/20080424/R-1305/R1305_1349_1.pdf (accessed on the 06 December 2011).

Gabaix, X., and D. Laibson (2006): "Shrouded Attributes, Consumer Myopia, and Information Suppression in Competitive Markets," The Quarterly Journal of Economics, 121(2), 505-540.

Holmström, B. (1999): "Managerial Incentive Problems: A Dynamic Perspective," Review of Economic Studies, 66(1), 169-182.

Inderst, R., and M. Ottaviani (2009a): "Misselling through Agents," The American Economic Review, 99(3), 883-908.

(2009b): "Sales Talk, Cancellation Terms, and the Role of Consumer Protection," http://didattica.unibocconi.it/mypage/upload/48832_20111118_025320 _REFUNDS.PDF.

IssacharofF, S. (1999): "Group Litigation of Consumer Claims: Lessons from the U.S. Experience," Texas International Law Journal, 34, 135-150.

Ivanov, M. (2011): "Information Revelation in Competitive Markets," Economic Theory, forthcoming.

Johnson, J. P., And D. P. Myatt (2006): "On the Simple Economics of Advertising, Marketing, and Product Design," The American Economic Review, 96(3), 756-784.

Judd, K. L., And M. H. Riordan (1994): "Price and Quality in a New Product Monopoly," The Review of Economic Studies, 61(4), 773-789.

Kartik, N. (2009): "Strategic Communication with Lying Costs," Review of Economic Studies, 76(4), 1359-1395. 
Kräkel, M., P. Nieken, and J. Przemeck (2008): "Risk Taking in WinnerTake-All Competition," Bonn Econ Discussion Paper bgse7_2008.

Lewis, T. R., and D. E. M. Sappington (1994): "Supplying Information to Facilitate Price Discrimination," International Economic Review, 35(2), 309-327.

LI, W. (2010): "Peddling Influence through Intermediaries," The American Economic Review, 100(3), 1136-1162.

Meyer, M. A., And J. Vickers (1997): "Performance Comparisons and Dynamic Incentives," The Journal of Political Economy, 105(3), 547-581.

Miceli, T. J. (1990): "Optimal Prosecution of Defendants Whose Guilt Is Uncertain," Journal of Law, Economics, and Organization, 6(1), 189-201.

Polinsky, A. M., and S. Shavell (2000): "The Economic Theory of Public Enforcement of Law," Journal of Economic Literature, 38(1), 45-76.

Posner, R. A. (2004): Frontiers of Legal Theory. Harvard University Press, Cambridge.

Shelegia, S. (2011): "Quality Choice of Experience Goods," http://homepage.univie.ac.at/sandro.shelegia/Personal/Research_files/quality choice.pdf.

Spiegler, R. (2006): "Competition over agents with boundedly rational expectations," Theoretical Economics, 1(2), 207-231.

Sunstein, C., R. Hastie, J. Payne, D. Schkade, and K. Viscusi (2002): Punitive Damages: How Juries Decide. University Of Chicago Press, Chicago.

Theil, H. (1971): Principles of Econometrics. North-Holland, Amsterdam.

Tsetlin, I., A. Gaba, and R. L. Winkler (2004): "Strategic Choice of Variability in Multiround Contests and Contests with Handicaps," Journal of Risk and Uncertainty, 29(2), 143-158.

Vickers, J. (2004): "Economics for Consumer Policy," in Proceedings of the British Academy, vol. 125. Oxford University Press, Oxford. 